\title{
Adaptive Estimation of the State of Charge for Lithium-Ion Batteries: Nonlinear Geometric Observer Approach
}

\author{
Wang, Y.; Fang, H.; Sahinoglu, Z.; Wada, T.; Hara, S.
}

\author{
TR2014-094 September 2014
}

\begin{abstract}
This paper considers the state of charge (SoC) and parameter estimation of lithium-ion batteries. Different from various prior arts, where estimation is based on local linearization of a nonlinear battery model, nonlinear geometric observer approach is followed to design adaptive observers for the SoC and parameter estimation based on nonlinear battery models. A major advantage of the proposed approach is the possibility to establish the exponential stability of the resultant error dynamics of state and parameter estimation. The proposed adaptive observers are shown to be robust with respect to unmodeled process uncertainties. Analysis also shows the design tradeoff between the convergence rate and the robustness of the estimation error dynamics with respect to the measurement noise. Simulation and experimental results validate the effectiveness and main advantages of the proposed approach. Error analysis is presented and explains the experimental results.
\end{abstract}

IEEE Transactions on Control Systems Technology

This work may not be copied or reproduced in whole or in part for any commercial purpose. Permission to copy in whole or in part without payment of fee is granted for nonprofit educational and research purposes provided that all such whole or partial copies include the following: a notice that such copying is by permission of Mitsubishi Electric Research Laboratories, Inc.; an acknowledgment of the authors and individual contributions to the work; and all applicable portions of the copyright notice. Copying, reproduction, or republishing for any other purpose shall require a license with payment of fee to Mitsubishi Electric Research Laboratories, Inc. All rights reserved. 



\title{
Adaptive estimation of the state of charge for Lithium-ion batteries: Nonlinear geometric observer approach
}

\author{
Yebin Wang, Member, IEEE, Huazhen Fang, Member, IEEE, Zafer Sahinoglu, Senior Member, IEEE, \\ Toshihiro Wada and Satoshi Hara
}

\begin{abstract}
This paper considers the State of Charge (SoC) and parameter estimation of Lithium-ion batteries. Different from various prior art, where estimation is based on local linearization of a nonlinear battery model, nonlinear geometric observer approach is followed to design adaptive observers for the $\mathrm{SoC}$ and parameter estimation based on nonlinear battery models. A major advantage of the proposed approach is the possibility to establish the exponential stability of the resultant error dynamics of state and parameter estimation. The proposed adaptive observers are shown to be robust with respect to unmodeled process uncertainties. Analysis also shows the design tradeoff between the convergence rate and the robustness of the estimation error dynamics with respect to the measurement noise. Simulation and experimental results validate the effectiveness and main advantages of the proposed approach. Error analysis is presented and explains the experimental results.
\end{abstract}

Index Terms-State of charge, batteries, adaptive estimation, geometric method, nonlinear systems.

\section{INTRODUCTION}

$\mathbf{L}$ ITHIUM-ION $\left(\mathrm{Li}^{+}\right)$batteries have been widely used in numerous applications including consumer electronics, automotive, and power tools, due to the high capacity but reduced size, superior power performance, and long cycle life [1]. Nowadays battery management systems (BMSs) are used to monitor the battery status and regulate the charging and discharging processes for real-time battery protection and performance improvement [2], [3]. An accurate state of charge (SoC) of the battery, usually defined as the percentage ratio of the present battery capacity to the maximum capacity, is a prerequisite to have a desirable BMS.

The SoC of a battery is difficult to measure, and its accurate estimation is known as a challenging task. Two straightforward SoC estimation methods are voltage translation and Coulomb

Manuscript received December 19, 2013; revised June 26, 2014; accepted August 24, 2014. Manuscript received in final form August 31, 2014. Date of publication XX, XX, 2014; date of current version XX, XX, 2014. Recommended by Associate Editor S. Varigonda.

Y. Wang and Z. Sahinoglu are with Mitsubishi Electric Research Laboratories, 201 Broadway, Cambridge, MA 02139, USA. (email: yebinwang@ieee.org, zafer@merl.com)

H. Fang is with the Department of Mechanical Engineering, University of Kansas, Lawrence, KS, and was an intern with Mitsubishi Electric Research Laboratories. (email: fang@ku.edu)

T. Wada and S. Hara are with the Advanced Technology R\&D Center, Mitsubishi Electric Corporation, 8-1-1, Tsukaguchi-honmachi, Amagasaki City, 661-8661, Japan. (email: Wada.Toshihiro@bx.MitsubishiElectric.co.jp, Hara.Satoshi@cb.MitsubishiElectric.co.jp) counting [3]. Both methods have limitations such as the former requires the battery to rest for a long period and cut off from the external circuit to measure the Open Circuit Voltage $(\mathrm{OCV})$, and the latter suffers cumulative integration errors and noise corruption.

Recently model-based approaches have attracted a lot of attention to improve the SoC estimation accuracy. For instance, equivalent circuit models (ECMs) and extended Kalman filter (EKF) type of approaches have been used extensively to estimate the $\mathrm{SoC}$ with approximate dynamic error bounds [4]-[6]. Nonlinear observer design approaches have also been applied to construct ECM-based nonlinear SoC estimators, e.g. sliding mode observer [7], adaptive model reference observer [8], Lyapunov-based observer [9] etc. ECM-based approaches have the benefit of simplicity and low computation but sacrifice physical meaning of model parameters thus may limit their uses for battery monitoring.

Electrochemical or physics-based models, for instance the pseudo two dimensional model [10], [11] and single particle models [12], [13], are derived from electrochemical principles which describe intercalation and diffusion of lithium ions and conservation of charge within a battery. Electrochemical models have the merit of ensuring each model parameter to retain a proper physical meaning; on the other hand, they take the form of nonlinear partial differential equations (PDEs) thus often necessitates model simplification or reduction for control and estimation purposes. A linear reduced-order electrochemical model is established in [14], to which the classical $\mathrm{KF}$ is employed for the SoC estimation. In [15], the EKF is implemented to estimate the $\mathrm{SoC}$ via a nonlinear ordinary differential equation (ODE) model obtained from PDEs by finite-difference discretization. The unscented Kalman filter (UKF) is used in [16] to avoid model linearization for more accurate SoC estimation. Rather than using the ODE model, nonlinear SoC estimators are also developed in [17], [18] through direct manipulation of PDEs.

Adaptive SoC estimation, which enables the SoC estimation with unknown model parameters, has been discussed for some ECMs and electrochemical models, e.g., [6], [19]-[22]. This paper is an extension of the work [22] by including alternative adaptive observer design, robustness and applicability analysis of the proposed adaptive observers, and experimental study. This paper makes new contributions to study of this topic by proposing nonlinear geometric observer approach 


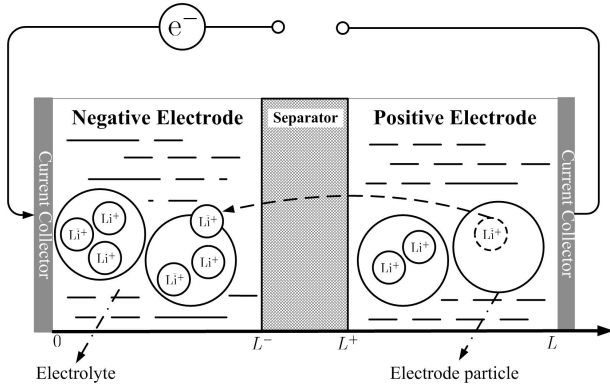

(a)

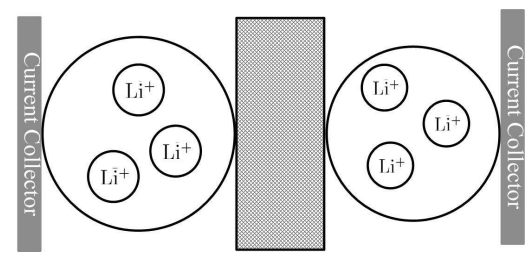

(b)

Fig. 1. Schematic characterization of battery dynamics: (a) the battery charging process; (b) the single-particle model.

for adaptive SoC estimation. The proposed nonlinear adaptive SoC estimators admit straightforward analysis of the resultant error dynamics. Specifically, for the case where no mismatch between the battery model and the physical process is present, the error dynamics are exponentially stable; with bounded model uncertainties, the error dynamics are input-to-state stable. Extensive analysis, and simulation and experimental results are provided to validate the methodology.

The rest of this paper is organized as follows. Section II introduces the working mechanism of $\mathrm{Li}^{+}$batteries and simplified battery models. Section III presents adaptive observers for the SoC and parameter estimation, and robustness analysis. Simulation and experiment results are provided in Section IV to verify the proposed design approach. The paper is concluded by Section V.

\section{Simplified BAtTERY MOdels}

This section includes a brief introduction of the working mechanism of $\mathrm{Li}^{+}$batteries, the single particle model (SPM) [2], and simplified models to which nonlinear geometric observer approach can be applied for the SoC estimation.

\section{A. The Working Mechanism of $\mathrm{Li}^{+}$Batteries}

A schematic visualization of $\mathrm{a} \mathrm{Li}^{+}$battery is presented in Fig 1(a). The positive electrode is typically made from $\mathrm{Li}$ compounds, e.g., $\mathrm{Li}_{q} \mathrm{Mn}_{2} \mathrm{O}_{4}$ and $\mathrm{Li}_{q} \mathrm{CoO}_{2}$. Small solid particles of the compounds are compressed to form a porous structure. Similarly, the negative electrode, usually containing graphite particles, is also porous. The interstitial pores at both electrodes provide intercalation space, where the $\mathrm{Li}^{+}$can be moved in and out and stored. The electrolyte contains free ions and is electrically conductive, where the $\mathrm{Li}^{+}$can be transported. The separator separates the electrodes apart. It allows the exchange of $\mathrm{Li}^{+}$from one side to the other, but prevents electrons from passing through. Electrons are thus forced to flow through the external circuit.

When the battery is being charged, $\mathrm{Li}^{+}$are extracted from particles at the positive electrode into the electrolyte, driven by reaction at the particle/electrolyte interface, and particles at the negative electrode absorbs $\mathrm{Li}^{+}$from the electrolyte. This process not only generates an influx of $\mathrm{Li}^{+}$within the battery, but also builds up a potential difference between the positive and negative electrodes. When it is reversed, the battery is discharging. The chemical reactions in the positive and negative electrodes are, respectively, described by

$$
\begin{gathered}
\mathrm{Li}_{q} \mathrm{Mn}_{2} \mathrm{O}_{4} \underset{\text { discharge }}{\stackrel{\text { charge }}{\rightleftharpoons}} \mathrm{Li}_{q-l} \mathrm{Mn}_{2} \mathrm{O}_{4}+l \mathrm{Li}^{+}+l \mathrm{e}^{-} \\
q \mathrm{Li}^{+}+q \mathrm{e}^{-}+\mathrm{C} \underset{\text { discharge }}{\stackrel{\text { charge }}{\rightleftharpoons}} \mathrm{Li}_{q} \mathrm{C}
\end{gathered}
$$

A rechargeable battery has various features including rate capacity effect (RCE), recovery effect (RE), and hysteresis effect (HE). A battery model capturing all these effects is important for accurate SoC and state of health estimation. It is however difficult to find practical models that interpret the RCE and the RE using electrochemical states [23]. A commonly used model for characterizing the electrochemical mechanism of $\mathrm{a} \mathrm{Li}^{+}$battery is presented in [10]. The model includes four quantities solid and electrolyte $\mathrm{Li}^{+}$concentration and potential as state variables, whose dynamics, e.g. material balance and charge balance, are captured by PDEs [13]. In addition, these state variables are coupled by a charge-transfer kinetic resistance at the particle surface which is given by the Butler-Volmer equations. Interested readers are referred to [2], [10], [13] for details.

\section{B. The Single Particle Model}

The single particle model (SPM) simplifies each electrode as a spherical particle with area equivalent to the active area of the electrode [24], [25]. The dynamics of electrolyte concentration and potential are ignored. Although unable to capture all electrochemical processes in batteries, the SPM reduces complexities in identification, estimation and control design to a large extent [15], [18]. To proceed further, a review of the SPM is provided, with the nomenclature shown in Table I.

Input and output of the battery: The external input to the battery is the current $I(t)$ with $I(t)<0$ for charge and $I(t)>$ 0 for discharge. The measured output of the battery is the terminal voltage, which is the potential difference between the two electrodes, and given by

$$
V(t)=\Phi_{s, p}(t)-\Phi_{s, n}(t) .
$$

Conservation of $\mathrm{Li}^{+}$in the electrode phase: The migration of $\mathrm{Li}^{+}$inside a particle is caused by the gradient-induced diffusion. Its dynamics can be modeled from the Fick's second law and given by

$$
\frac{\partial c_{s, j}(r, t)}{\partial t}=\frac{1}{r^{2}} \frac{\partial}{\partial r}\left(D_{s, j} r^{2} \frac{\partial c_{s, j}(r, t)}{\partial r}\right),
$$




\begin{tabular}{|ll|}
\hline Variables \\
$\Phi_{s}$ & electric potential in the solid electrode \\
$\Phi_{e}$ & electric potential in the electrolyte \\
$c_{S}$ & concentration of $\mathrm{Li}^{+}$in the solid electrode \\
$c_{s s}$ & concentration of $\mathrm{Li}^{+}$at a particle's spherical surface \\
$J$ & molar flux of $\mathrm{Li}^{+}$at the particle's surface \\
$J_{0}$ & exchange current density \\
$\eta$ & overpotential of reaction in the cell \\
$U$ & open-circuit potential \\
$I$ & external circuit current \\
$V$ & terminal voltage \\
$x$ & original system state (state of charge) \\
$y$ & measured terminal voltage of a battery \\
$\xi$ & transformed system state (terminal voltage of a battery) \\
& \\
Physical parameters \\
$D_{s}$ & diffusion coefficient of Li in the solid electrode \\
$r$ & radius of the spherical particle \\
$F$ & Farady's constant \\
$S$ & specific interfacial area \\
$T$ & temperature of the cell \\
$\alpha_{a}$ & anodic charge transport coefficient \\
$\alpha_{c}$ & cathodic charge transport coefficient \\
$R$ & universal gas constant \\
$R_{C}$ & phase resistance \\
$R_{f}$ & film resistance of the solid electrolyte interphase \\
$\alpha$ & charge coefficient \\
$\beta$ & parameters in the OCV-SoC curve \\
$\gamma_{1}$ & battery internal resistance \\
Subscripts \\
$S$ & solid electrode phase \\
$e$ & electrolyte phase \\
$n$ & negative electrode \\
$p$ & positive electrode \\
& $n$ or $p$ \\
\hline
\end{tabular}

DEFINITIONS AND NOMENCLATURE.

with the following initial and boundary conditions

$$
c_{s, j}(r, 0)=c_{s}^{0},\left.\quad \frac{\partial c_{s, j}}{\partial r}\right|_{r=0}=0,\left.\quad \frac{\partial c_{s, j}}{\partial r}\right|_{r=\bar{r}_{j}}=-\frac{1}{D_{s, j}} J_{j} .
$$

It is noted that $J_{j}$ is the molar flux at the electrode/electrolyte interface of a single particle. When $j=n$ and $p$, respectively,

$$
J_{n}(t)=\frac{I(t)}{F S_{n}}, \quad J_{p}(t)=-\frac{I(t)}{F S_{p}} .
$$

Electrochemical kinetics: The molar flux $J_{j}$ is governed by the Butler-Volmer equation:

$$
J_{j}(t)=\frac{J_{0, j}}{F}\left[\exp \left(\frac{\alpha_{a} F}{R T} \eta_{j}(t)\right)-\exp \left(-\frac{\alpha_{c} F}{R T} \eta_{j}(t)\right)\right],
$$

where $\eta_{j}(t)=\Phi_{s, j}(t)-\Phi_{e, j}(t)-U\left(c_{s s, j}(t)\right)-F R_{f, j} J_{j}(t)$. The electrolyte phase can be represented by a resistor $R_{c, j}$ in the SPM, implying $\Phi_{e, j}$ can be expressed as $\Phi_{e, j}(t)=R_{c, j} I(t)$. Hence, $\eta_{j}$ becomes

$$
\eta_{j}(t)=\Phi_{s, j}(t)-U\left(c_{s s, j}(t)\right)-F \bar{R}_{j} J_{j}(t),
$$

where $\bar{R}_{j}=S_{j} R_{c, j}+R_{f, j}$.

The SPM is represented by (1)-(3), in which $I$ is the external input, $c_{s, j}$ and $\Phi_{s, j}$ are the variables showing the battery status, and $V$ is the model output. The SPM could not fully capture the RCE since assumptions used in its derivation are merely valid at low charge-discharge rates.

\section{A Simplified Battery Model}

Nonlinear geometric observer approach assumes a system represented by ODEs, thus the SPM should be further simplified. Many techniques have been proposed to meet this purpose. One way is to introduce a volume average concentration as a state variable thus eliminate the two diffusion PDEs of $\mathrm{Li}^{+}$concentration in particles.

Average $\mathrm{Li}^{+}$concentration in the electrode phase: Throughout the paper the average concentration of $\mathrm{Li}^{+}$in the particle is treated as the measure of the battery capacity, or equivalently, the SoC. For an electrode particle, it is defined as

$$
c_{s, j}^{\mathrm{avg}}(t)=\frac{1}{\Omega} \int_{\Omega} c_{s, j}(r, t) \mathrm{d} \Omega,
$$

where $\Omega$ denotes the volume of the particle sphere. From (2), it is obtained that

$$
\begin{aligned}
\dot{c}_{s, j}^{\mathrm{avg}}(t) & =\frac{1}{\Omega} \int_{\Omega} \frac{\partial c_{s, j}(r, t)}{\partial t} \mathrm{~d} \Omega \\
& =\frac{1}{\Omega} \int_{\Omega} \frac{1}{r^{2}} \frac{\partial}{\partial r}\left(D_{s, j} r^{2} \frac{\partial c_{s, j}(r, t)}{\partial r}\right) \mathrm{d} \Omega \\
& =\left.\varepsilon_{j} D_{s, j} \frac{\partial c_{s, j}(r, t)}{\partial r}\right|_{r=\bar{r}_{j}},
\end{aligned}
$$

where $\varepsilon_{j}$ is a constant coefficient. Depending on the electrode polarity, (6) splits into

$$
\begin{aligned}
& \dot{c}_{s, n}^{\mathrm{avg}}(t)=-\frac{\varepsilon_{n}}{F S_{n}} I(t), \\
& \dot{c}_{s, p}^{\mathrm{avg}}(t)=\frac{\varepsilon_{p}}{F S_{p}} I(t) .
\end{aligned}
$$

It is noted from (7)-(8) that $\dot{c}_{s, j}^{\text {avg }}$ is linearly proportional to the input current $I$. In other words, $c_{s, j}^{\mathrm{avg}}$ is equal to the initial value $c_{s, j}^{\mathrm{avg}}(0)$ plus integration of $I$ over time. This illustrates that the change of SoC depends linearly on $I$ as a result of $c_{s, j}^{\text {avg }}$ indicating SoC. Such a relationship has not only been presented for electrochemical models, e.g., [14], but has also been justified in ECMs, e.g., [5], [26] and the references therein.

Terminal voltage: Suppose there exists a function $\varphi$ such that $c_{s s, j}(t)=\varphi\left(c_{s, j}^{\mathrm{avg}}(t)\right)$ and define $\bar{U}=U \circ \varphi$, where ' $\circ$ ' denotes composition of two functions. Using (4), (1) becomes

$V(t)=\bar{U}\left(c_{s, p}^{\mathrm{avg}}(t)\right)-\bar{U}\left(c_{s, n}^{\mathrm{avg}}(t)\right)+\eta_{p}(t)-\eta_{n}(t)+\left(\bar{R}_{p}-\bar{R}_{n}\right) I(t)$.

With $\alpha_{a}=\alpha_{c}=0.5$, it follows from (3) that

$$
\begin{aligned}
& \eta_{n}(t)=\frac{2 R T}{F} \sinh ^{-1}\left(\frac{J_{n}(t) F}{2 J_{0, n}}\right)=\frac{2 R T}{F} \sinh ^{-1}\left(\frac{\varepsilon_{n} I(t)}{2 J_{0, n}}\right), \\
& \eta_{p}(t)=\frac{2 R T}{F} \sinh ^{-1}\left(\frac{J_{p}(t) F}{2 J_{0, p}}\right)=\frac{2 R T}{F} \sinh ^{-1}\left(-\frac{\varepsilon_{p} I(t)}{2 J_{0, p}}\right) .
\end{aligned}
$$

Thus $V(t)$ becomes

$$
\begin{aligned}
V(t) & =\bar{U}\left(c_{s, p}^{\mathrm{avg}}\right)-\bar{U}\left(c_{s, n}^{\mathrm{avg}}\right) \\
& +\frac{2 R T}{F}\left[\sinh ^{-1}\left(-\frac{\varepsilon_{p} I(t)}{2 J_{0, p}}\right)-\sinh ^{-1}\left(\frac{\varepsilon_{n} I(t)}{2 J_{0, n}}\right)\right] \\
& +\left(\bar{R}_{p}-\bar{R}_{n}\right) I(t) .
\end{aligned}
$$


As such, $V(t)$ consists of two parts. The first is the open-circuit voltage (OCV) that relies on $\bar{U}\left(c_{s, j}^{\mathrm{avg}}\right)$, and the second is the direct feedthrough from $I$ to $V$.

System (7)-(9) provides a concise characterization of the battery dynamics. As pointed out in [18], the SPM implies the conservation of Lithium ions within two particles, or equivalently, that $c_{s s, p}^{a v g}(t)$ can be represented as a linear function of $c_{s s, n}^{a v g}(t)$. This fact allows the reduction of (7)-(8) into one differential equation. With $c_{s s, n}^{a v g}(t)$ as the independent variable, the battery dynamics is captured by (7) and (9). As aforementioned, $c_{s s, n}^{a v g}(t)$ is arguably equivalent to the SoC. Denoting the SoC by a state $x \in[0,1]$, and defining the input $u$ and the output $y$ of the model as the charge current $I$ and the terminal voltage $V$ of the battery, respectively, we have the battery model as follows

$$
\begin{aligned}
& \dot{x}=-\alpha u, \\
& y=h(x, \beta)+g(u, \gamma),
\end{aligned}
$$

where $\alpha, \beta=\left(\beta_{1}, \beta_{2}, \beta_{3}\right)$, and $\gamma=\left(\gamma_{0}, \gamma_{1}, \gamma_{2}, \gamma_{3}\right)$ are unknown parameters, $h(x, \beta)$, the part containing $\bar{U}$ in (9), takes the parametric form of $h(x)=\beta_{1} \ln \left(x+\beta_{2}\right)+\beta_{3}$, and $g(u, \gamma)$ corresponding to the part involving $I$ in (9) is expressed as $g(u, \gamma)=\gamma_{0}\left[\sinh ^{-1}\left(\gamma_{2} u\right)-\sinh ^{-1}\left(\gamma_{3} u\right)\right]+\gamma_{1} u$, where $\gamma_{i}$ for $i=0,1,2,3$ are from (9).

Due to the negligence of diffusion in solid particles, the battery model (10) have limited capability to represent the RCE, the RE and the HE. This paper however uses the battery model (10) mainly for illustration purpose, i.e., to demonstrate how nonlinear geometric observer approach can be followed to perform adaptive SoC estimation.

Remark 2.1: The thermodynamics of batteries are not covered by the SPM and the simplified model (10). Ignoring thermodynamics however might not impose significant restriction to the applicability of the proposed approach. Loosely speaking, the effect of thermodynamics on both models can be compensated by making parameters time-varying. Adaptive state estimation based on (10) is possible for cases where parameters are either constant or time-varying but with bounded time derivative [27]. If the thermodynamics evolves in a much slower time scale than the estimation error dynamics, the adaptive estimator generally can track the resultant slow timevarying parameters and original system state with reasonable accuracy.

\section{A Switched Battery Model}

An intuitive generalization of the model (10) can be done by addressing the HE in the OCV-SoC relationship, which is characterized by $h(x, \beta)$. We consider the following switched battery model

$$
\begin{aligned}
& \dot{x}=-\alpha u, \\
& y= \begin{cases}h(x, \beta)+g(u, \gamma), & u>0, \\
h(x, \bar{\beta})+g(u, \gamma), & u \leq 0,\end{cases}
\end{aligned}
$$

where $\bar{\beta}=\left(\bar{\beta}_{1}, \bar{\beta}_{2}, \bar{\beta}_{3}\right)$ is constant. Here $h(x, \beta)$ and $h(x, \bar{\beta})$ parameterize the discharge and charge OCV-SoC curves, respectively. As will be shown later, nonlinear geometric observer approach can also be applied to the switched model (11) for adaptive SoC estimation. The resultant SoC estimation is more accurate than that based on the model (10).

\section{MAin Results}

Similar to [21], a two-stage approach is used to perform adaptive SoC estimation:

- Stage 1: As $h(\cdot)$ represents the OCV, it is determined using the SoC-OCV data set to identify parameters $\beta$ for the battery model (10), or to identify parameters $\beta$ and $\bar{\beta}$ for the battery model (11).

- Stage 2: After $\beta$ and/or $\bar{\beta}$ is identified, the state $x$ and parameters $\alpha, \gamma$ are estimated simultaneously by nonlinear geometric observer approach.

The identification in Stage 1 has been well-studied in literatures. As an example, one can formulate it as a nonlinear least squares data fitting problem and solve the resultant nonlinear programming problem. Interested readers are referred to [28], [29] for further details. This paper focuses on the problem in Stage 2, where parameters $\beta$ are treated as known constants, and the state and parameter estimation is performed using nonlinear geometric observer approach.

We first define a local adaptive observer for a general system

$$
\begin{aligned}
& \dot{\zeta}=f(\zeta, \Theta), \\
& y=h(\zeta),
\end{aligned}
$$

where $\zeta \in \mathbb{R}^{n}$ is the system state, $f: \mathbb{R}^{n} \times \mathbb{R}^{m} \rightarrow \mathbb{R}^{n}$ is a smooth vector field, $\Theta \in \mathbb{R}^{m}$ is the unknown parameter vector, and $h: \mathbb{R}^{n} \rightarrow \mathbb{R}^{p}$ is a vector of smooth functions. Here the notation $h$ is abused, which should not incur any confusion given the context.

Definition 3.1: [30] A local adaptive observer for system (12) with the presence of the unknown parameter $\Theta$ in $f$ is a finite dimensional system

$$
\begin{array}{ll}
\dot{w}=\alpha_{1}(w, \hat{\Theta}, y(t)), & w \in \mathbb{R}^{r}, r \geq n, \\
\dot{\hat{\Theta}}=\alpha_{2}(w, \hat{\Theta}, y(t)), & \hat{\Theta} \in \mathbb{R}^{m}, \\
\hat{\zeta}=\alpha_{3}(w, \hat{\Theta}, y(t)), & \hat{\zeta} \in \mathbb{R}^{n}
\end{array}
$$

driven by $y(t)$, such that for every $\zeta(0) \in \mathbb{R}^{n}, w(0) \in U_{w} \subset$ $\mathbb{R}^{r}, \hat{\Theta}(0) \in U_{\Theta} \subset \mathbb{R}^{m}$, where $U_{w}, U_{\Theta}$ are the neighborhoods of $\zeta(0), \Theta$ respectively, for any value of the unknown parameter $\Theta$ and for any bounded $\|\zeta(t)\|, \forall t \geq 0$ :

1) $\|w(t)\|,\|\hat{\Theta}(t)\|$ and $\|\zeta(t)-\hat{\zeta}(t)\|$ are bounded, $\forall t \geq 0$.

2) $\lim _{t \rightarrow \infty}\|\zeta(t)-\hat{\zeta}(t)\|=0$.

In Definition 3.1, a local adaptive observer is defined for a system where the output function $h$ does not have explicit dependence on unknown parameters. This is without loss of generality because one can introduce a parameter dependent state transformation to put a general system into the form (12).

\section{A. Nonlinear Geometric Observer Approach}

We first define some notation. Given a $\mathrm{C}^{\infty}$ vector field $f$ : $\mathbb{R}^{n} \rightarrow \mathbb{R}^{n}$, and a $\mathrm{C}^{\infty}$ function $h: \mathbb{R}^{n} \rightarrow \mathbb{R}$, the function $L_{f} h(x)=$ $\frac{\partial h(x)}{\partial x} f$ is the Lie derivative of $h(x)$ along $f$. Repeated Lie 
derivatives are defined as $L_{f}^{k} h(x)=L_{f}\left(L_{f}^{k-1} h(x)\right), k \geq 1$ with $L_{f}^{0} h(x)=h(x)$.

Nonlinear geometric observer approach generally requires a system in some forms which enable the simplification of observer design and establishment of the error dynamics stability. Typically, the first step for nonlinear geometric observer approach is to put the original system into an observable form by change of coordinates. As a next step starting from the observable form, various techniques, for instance output transformation [31], [32], state transformation [31], [33], time scale transformation [34]-[36], dynamics extension [37], and approximate transformation [38], can be considered to further simplify the system dynamics. The transformed system typical takes a certain special structure, e.g. observer form [31], [33], [39], block triangular forms [40]-[43], time scaled observer forms [34], [36], [44], and adaptive observer forms [45]-[47] etc. As the final step, observer design for a system admitting special coordinates can be readily performed, and usually have properties: reduced design complexity, straightforward determination of the observer gain, and simplified estimation error dynamics. Although nonlinear geometric observer approach allows systematic and simple observer designs, and yields stable estimation error dynamics, it suffers from limited range of applicability.

For a locally uniformly observable single input single output (SISO) system,

$$
\begin{aligned}
& \dot{\zeta}=f(\zeta)+g(\zeta) u, \\
& y=h(\zeta),
\end{aligned}
$$

where $u \in \mathbb{R}$ and $h: \mathbb{R}^{n} \rightarrow \mathbb{R}$, the observable form exists and is uniquely defined as follows [48]

$$
\dot{x}=\left[\begin{array}{c}
x_{2} \\
\vdots \\
L_{f}^{n} h(\zeta)
\end{array}\right]+\left[\begin{array}{c}
g_{1}\left(x_{1}\right) \\
\vdots \\
g_{n}(x),
\end{array}\right] u,
$$

where $x=\Phi(\zeta)=\left(h(\zeta), \cdots, L_{f}^{n-1} h(\zeta)\right)^{T}$ and $\zeta=\Phi^{-1}(x)$. Observability ensures that $x$ defines new coordinates. For a multi-output system, the observable form is not uniquely defined due to the non-uniqueness of the observability definitions and observability indices [40], [42]. Readers are referred to [30], [40], [42], [48], [49] for details about observability and observable forms. One can readily verify that the battery model (10), SISO with one state, is locally observable. The identifiability of parameters can be verified by performing observability analysis on the augmented system which includes (10) and the following dynamics

$$
\begin{aligned}
\dot{\alpha} & =0, \\
\dot{\gamma}_{1} & =0 .
\end{aligned}
$$

Readers are referred to [50] for detailed observability and identifiability analysis.

Earlier work on adaptive observer with nonlinear parameterizations includes a local adaptive observer [51] based on a nonlinearly parameterized observer form, and a semi-global adaptive observer [47] etc. The battery model (10) does not admit the nonlinearly parameterized observer form in [51].
This paper mainly performs adaptive $\mathrm{SoC}$ estimation based on work [47]. The fact that this paper applies results of work [47] however shall not prevent one from applying other relevant works. For an SISO system, work [47] considered adaptive observer design for a class of nonlinearly parameterized system on the basis of the following form

$$
\begin{aligned}
& \dot{z}=A z+\varphi(z, u, \Theta), \\
& y=C z,
\end{aligned}
$$

where $(A, C)$ is in Brunovsky observer form, $z=\left(z_{1}, \ldots, z_{n}\right)^{T}=\in \mathbb{R}^{n}$ is the state vector, $\Theta \in \mathbb{R}^{m}$ is the unknown parameter vector, $u \in \mathbb{R}^{s}$ is the input vector, and $\varphi(z, u, \Theta)$ takes the following form

$$
\varphi(z, u, \Theta)=\left[\begin{array}{c}
\varphi_{1}\left(z_{1}, u, \Theta\right), \\
\vdots \\
\varphi_{n}(z, u, \Theta)
\end{array}\right] .
$$

Note that $\varphi(z, u, \Theta)$ has triangular dependence on $z$ to enable high gain observer design [48], [52].

Given a nonlinear system in the form (14), work [47] proposed the following dynamical system

$$
\begin{aligned}
\dot{\hat{z}} & =A \hat{z}+\hat{\varphi}-\theta \Delta_{\theta}^{-1}\left(S^{-1}+\Upsilon P \Upsilon^{T}\right) C^{T} K(y-\hat{y}), \\
\hat{\hat{\Theta}} & =\theta P \Upsilon^{T} C^{T} K(y-\hat{y}), \\
\dot{\Upsilon} & =-\theta\left(A-S^{-1} C^{T} C\right) \Upsilon+\Delta_{\theta} \frac{\partial \hat{\varphi}}{\partial \hat{\Theta}} \\
\dot{P} & =-\theta P \Upsilon^{T} C^{T} C \Upsilon P+\theta P, \quad P(0)=P_{0}>0,
\end{aligned}
$$

where $\theta$ is a positive constant, $\hat{\varphi}=\varphi(\hat{z}, u, \hat{\Theta}), \hat{y}=C \hat{z}$, $\Delta_{\theta}=\operatorname{diag}\left[1, \frac{1}{\theta}, \ldots, \frac{1}{\theta^{n-1}}\right]$, and $S$ is the unique solution of the algebraic Lyapunov equation

$$
S+A^{T} S+S A-C^{T} C=0 .
$$

To show the system (15) is a semi-global adaptive observer of the original system (14), the following technical assumptions are assumed.

Assumption 3.2: [47, Assum. (A1)] The state $z(t)$, the control $u(t)$ and the unknown parameters $\Theta$ are bounded, i.e., $z(t) \in Z, u(t) \in U$ for $t \geq 0$ and $\Theta \in \Omega$ where $Z \subset R^{n}, U \subset R^{s}$ and $\Omega \subset \mathbb{R}^{m}$.

Assumption 3.3: [47, Assum. (A2')] The function $\varphi(z, u, \Theta)$ is Lipschitz with respect to $z$ and $\Theta$, uniformly in $u$ where $(z, u, \Theta) \in Z \times U \times \Omega$.

Assumption 3.4: [47, Assum. (A3')] The nonlinear parametrization function $\varphi(z, u, \cdot)$ is one to one from $\mathbb{R}^{m}$ into $\mathbb{R}^{m}$.

Assumption 3.5: [47, Assum. (A4')] The inputs $u$ are such that for any trajectory of system (15) starting from $(\hat{z}(0), \hat{\Theta}(0)) \in Z \times \Omega$, the matrix $C \Upsilon$ is persistently exciting, i.e., $\exists \delta_{1}, \delta_{2}>0 ; \exists T>0 ; \forall t \geq 0$,

$$
\delta_{1} I_{n} \leq \int_{t}^{t+T} \Upsilon^{T} C^{T} C \Upsilon \mathrm{d} \tau \leq \delta_{2} I_{n}
$$

where $I_{n} \in \mathbb{R}^{n \times n}$ is the identity matrix.

[47, Thm. 4.2] established that the system (15) is a semiglobal adaptive observer of the original system (14). For completeness of this paper, we recite the theorem as follows 
Theorem 3.6: [47, Thm. 4.2] under assumptions (A1),(A2'),(A3') and (A4'), system (15) is an adaptive observer for system (14) with an exponentially error convergence for relatively high values of $\theta$.

\section{B. System Transformation}

Following the nonlinear geometric observer approach in Section III-A, we first transform the battery model (10) into certain forms which facilitate adaptive observer design. To simplify the presentation, this paper assumes $g(u, \gamma)$ in system (10) has a linear parametrization. Specifically, consider the following battery model

$$
\begin{aligned}
& \dot{x}=\alpha u, \\
& y=\beta_{1} \log \left(x+\beta_{2}\right)+\beta_{3}+\gamma_{1} u,
\end{aligned}
$$

where $x$ is the SoC of a battery, $\beta_{i}$ are known parameters, and $\gamma_{1}, \alpha$ are unknown parameters.

Remark 3.7: Assuming $g(u, \gamma)=\gamma_{1} u$ is mainly to simplify the state transformation and the expression of the resultant transformed system, and without loss of generality. With the original $g(u, \gamma)$, the state transformation is taken as follows

$$
\xi(x, u, \gamma)=\beta_{1} \log \left(x+\beta_{2}\right)+\beta_{3}+g(u, \gamma),
$$

which is a diffeomorphism over $\mathbb{R}^{+}$. The inverse state transformation can be similarly computed as follows

$$
x+\beta_{2}=\exp \left(\frac{y-\beta_{3}-g(u, \gamma)}{\beta_{1}}\right) .
$$

The procedure followed in the sequel to develop adaptive observers is therefore still applicable for the original $g(u, \gamma)$ case. Simplifying $g(u, \gamma)$ as $\gamma_{1} u$ can also be justified by its practical usefulness. That is: because $\gamma_{2}, \gamma_{3}$ are usually small positive constants, the ignored term $\gamma_{0}\left(\operatorname{asinh}\left(\gamma_{2} u\right)-\operatorname{asinh}\left(\gamma_{3} u\right)\right)$ can be approximated by a linear function of $u$, thus can be lumped into the term $\gamma_{1} u$. For a particular battery tested in the experiment, $\gamma_{1}$ is in the order of milliohms (m-Ohm). We therefore take $\gamma_{2}$ and $\gamma_{3}$ at the same order, i.e., $\gamma_{2}=1 e-3, \gamma_{3}=$ $2 e-3$, and examine the plot of the ignored term versus $u$. As shown in Figure 2, the ignored term is almost linear in $u$ over a wide range of input current: from -50Amps to 50Amps. Note that the tested battery has a nominal capacity $5 \mathrm{Amp}$ hours (Ah) and the 50Amps current is quite large (10C). A less rigorous interpretation of the aforementioned analysis is that parameters $\gamma_{i}, 0 \leq i \leq 3$ are closely coupled and almost indistinguishable, and can be lumped into one parameter.

Putting (16) into observable form (13) with unknown parameterizations requires the following parameter dependent state transformation

$$
\xi\left(x, u, \gamma_{1}\right)=\beta_{1} \log \left(x+\beta_{2}\right)+\beta_{3}+\gamma_{1} u,
$$

where $\xi$ is the new state variable and denotes the terminal voltage of the battery. We have

$$
\dot{\xi}=\beta_{1} \frac{\alpha}{x+\beta_{2}} u+\gamma_{1} \dot{u},
$$

where $x+\beta_{2}$ can be solved as

$$
x+\beta_{2}=\exp \left(\frac{y-\beta_{3}-\gamma_{1} u}{\beta_{1}}\right) \text {. }
$$

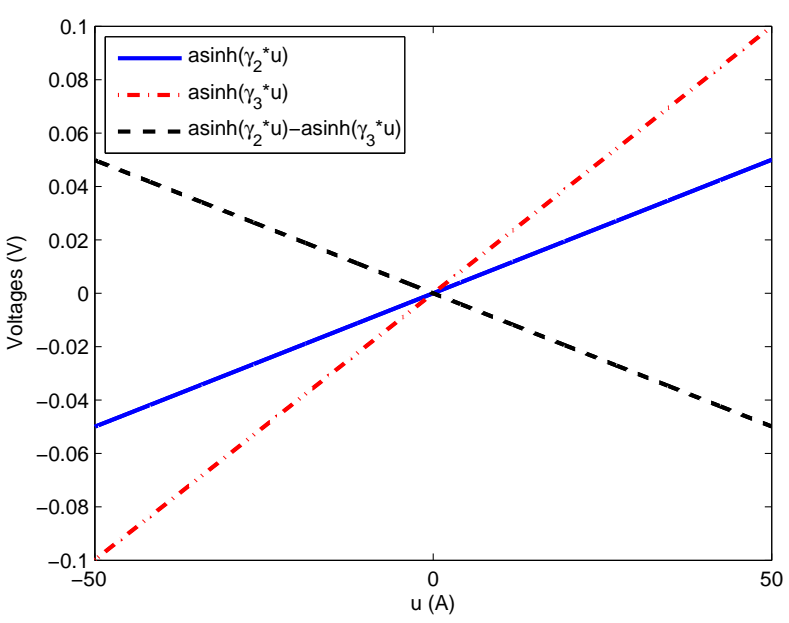

Fig. 2. Curves of $\operatorname{asinh}\left(\gamma_{2} u\right), \operatorname{asinh}\left(\gamma_{3} u\right)$ and $\operatorname{asinh}\left(\gamma_{2} u\right)-\operatorname{asinh}\left(\gamma_{3} u\right)$

We rearrange the transformed system and have

$$
\begin{aligned}
& \dot{\xi}=\phi(y, u, \gamma, \alpha)+\gamma_{1} \dot{u}, \\
& y=\xi
\end{aligned}
$$

where

$$
\phi(\cdot)=\beta_{1} \alpha \exp \left(\frac{\beta_{3}+\gamma_{1} u-y}{\beta_{1}}\right) u .
$$

Remark 3.8: The SoC, represented by $x$, is always positive, also $x+\beta_{2}$ has to be positive if the model (16) is valid. One can verify that the state transformation (17) is a diffeomorphism over $x \in \mathbb{R}^{+}$, i.e., the state transformation (17) is well-defined in the domain where the model (16) is physically meaningful.

The system (18) in the observable coordinates has nonlinear parameterizations of $\alpha$ and $\gamma_{1}$. The transformed system (18) is already in the form (14), and thus no further transformation is required to perform adaptive observer design.

\section{An Adaptive Observer}

The transformed system (18) is in the form of (14), where

$$
\varphi\left(y, u, \dot{u}, \gamma_{1}, \alpha\right)=\beta_{1} \alpha \exp \left(\frac{\beta_{3}+\gamma_{1} u-y}{\beta_{1}}\right) u+\gamma_{1} \dot{u} .
$$

The transformed system (18) is linearly parameterized by $\alpha$ but nonlinearly parameterized by $\gamma_{1}$. Given all assumptions in [47] satisfied for system (18), we could perform adaptive observer design for adaptive SoC estimation.

For a physical battery, the state $\xi$ or equivalently the battery's terminal voltage $y$, the external current input $u$ and its time derivative $\dot{u}$, and model parameters $\gamma_{1}, \alpha$ are bounded in a compact set $\mathscr{D} \subset \mathbb{R}^{5}$. Assumption [47, Assum. (A1)] is satisfied. Given $u, \dot{u}, y$ are bounded by a compact set $\mathscr{D}$, the smooth function $\varphi$ is Lipschitz with respect to $\rho=\left(\alpha, \gamma_{1}\right)^{T}$ and uniformly in $u, \dot{u}, y$, i.e., given any 3 -tuple $(y, u, \dot{u}) \in \mathscr{D}$, there exists a constant $L$ such that the following inequality holds

$$
\left\|\varphi\left(y, u, \dot{u}, \rho^{1}\right)-\varphi\left(y, u, \dot{u}, \rho^{2}\right)\right\| \leq L\left\|\rho^{1}-\rho^{2}\right\|,
$$

for any $\rho^{1}=\left(\alpha^{1}, \gamma_{1}^{1}\right), \rho^{2}=\left(\alpha^{2}, \gamma_{1}^{2}\right) \in \Omega \subset \mathscr{D}$. 
Remark 3.9: Using the state transformation (17) also implies the differentiability of $\xi$, which requires the input current $u$ is differentiable. In addition, $\dot{u}$ has to be bounded in order to satisfy Assumption 3.2. These conditions on the input current $u$ sound restrictive from theoretical perspective, but are not really a restriction in reality. Performing observer design based on system dynamics having explicit dependence on $\dot{u}$ however indeed makes the observer sensitive to the measurement noise.

Assumption [47, Assum. (A3')] is however not satisfied because given a fixed 3 -tuple $(y, u, \dot{u})$, one can easily find two sets of parameters $\rho^{1}$ and $\rho^{2}$ such that $\varphi\left(y, u, \dot{u}, \rho^{1}\right)=$ $\varphi\left(y, u, \dot{u}, \rho^{2}\right)$. Notice that Assumption [47, Assum. (A3')] is not explicitly used to show the convergence, and the proof of Theorem [47, Thm. 4.2] merely relies on Assumptions (A1'),(A2'),(A4'). We may still be able to have an adaptive observer as long as Assumption [47, Assum. (A4')] is verified.

We consider the following system

$$
\begin{aligned}
& \dot{\hat{\xi}}=\theta(y-\hat{y})+\hat{\varphi}+\theta^{2} \Upsilon P \Upsilon^{T}(y-\hat{y}), \quad \hat{\xi}(0)=\xi_{0} \in Z, \\
& \dot{\Upsilon}=-\theta \Upsilon+\frac{\partial \hat{\varphi}}{\partial \hat{\rho}}, \quad \Upsilon(0)=\Upsilon_{0} \in \mathbb{R}^{2}, \\
& \dot{\hat{\rho}}=\theta P \Upsilon^{T}(y-\hat{y}), \quad \hat{\rho}(0)=\hat{\rho}_{0} \in \Omega, \\
& \dot{P}=-\theta P \Upsilon^{T} \Upsilon P+\theta P, \quad P(0)=P_{0} \in \mathbb{R}^{2 \times 2},
\end{aligned}
$$

where $\hat{\rho}=\left[\hat{\alpha}, \hat{\gamma}_{1}\right]^{T}$,

$$
\hat{\varphi}=\varphi(y, u, \dot{u}, \operatorname{sat}(\hat{\rho})),
$$

$\theta$ is a sufficiently large positive constant, $\hat{\xi}_{0}$ is constant, $\Upsilon_{0}$ is bounded and constant, $P_{0}$ is positive definite, and

$$
\frac{\partial \hat{\varphi}}{\partial \hat{\rho}}=\left(\left.\frac{\partial \varphi(y, u, \dot{u}, \hat{\rho})}{\partial \hat{\rho}}\right|_{\hat{\rho}=\operatorname{sat}(\hat{\rho})}\right)^{T} .
$$

The notation sat $(\cdot)$ performs an element-wise saturation if its argument is a vector, for instance,

$$
\operatorname{sat}(\hat{\rho})=\left[\begin{array}{l}
\operatorname{sat}(\hat{\alpha}) \\
\operatorname{sat}\left(\hat{\gamma}_{1}\right)
\end{array}\right] .
$$

If its argument is a scalar, the sat $(\cdot)$ operation is exemplified by the $\operatorname{sat}(\hat{\alpha})$ as follows

$$
\operatorname{sat}(\hat{\alpha})= \begin{cases}\underline{\alpha}, & \text { if } \hat{\alpha} \leq \underline{\alpha}, \\ \hat{\alpha}, & \text { if } \hat{\alpha} \in(\underline{\alpha}, \bar{\alpha}), \\ \bar{\alpha}, & \text { if } \hat{\alpha} \geq \bar{\alpha},\end{cases}
$$

where $\underline{\alpha}$ and $\bar{\alpha}$ are the lower and upper bounds of $\alpha$. Similarly, $\operatorname{sat}(\hat{\xi})$ and $\operatorname{sat}\left(\hat{\gamma}_{1}\right)$ saturate $\hat{\xi}$ and $\hat{\gamma}_{1}$, based on bounds of $\xi$ and $\gamma_{1}$, respectively. Given $\hat{\varphi}=\varphi(y, u, \dot{u}$, sat $(\hat{\rho}))$, Assumption 3.3, when applied to the system (18), reads: the function $\varphi(y, u, \dot{u}, \rho)$ is Lipschitz with respect to $\rho$, uniformly in $y, u$ and $\dot{u}$, i.e., (19) holds for any $\rho^{1}, \rho^{2} \in \Omega$ and $(y, u, \dot{u}) \in \mathscr{D}$.

Remark 3.10: The term $\hat{\varphi}$ can be taken as

$$
\hat{\varphi}=\varphi(\operatorname{sat}(\hat{\xi}), u, \dot{u}, \operatorname{sat}(\hat{\rho})),
$$

and Assumption 3.3 is modified accordingly: the function $\varphi(\xi, u, \dot{u}, \rho)$ is Lipschitz with respect to $(\xi, \rho)$, uniformly in $u$ and $\dot{u}$, i.e., there exists a constant $L$ such that the following inequality holds

$$
\left\|\varphi\left(u, \dot{u}, X^{1}\right)-\varphi\left(u, \dot{u}, X^{2}\right)\right\| \leq L\left\|X^{1}-X^{2}\right\|,
$$

for any $X^{1}=\left(\xi^{1}, \rho^{1}\right), X^{2}=\left(\xi^{2}, \rho^{2}\right) \in Z \times \Omega$.

As pointed out in [47], the saturation of $\hat{\xi}$ and $\hat{\rho}$ in (21) and (22) is required to apply the Lipschitz conditions, e.g. (19) and (23), which is critical in the stability proof. For the case where $\hat{\varphi}$ is given by (22), although the original state and parameters are bounded in the domain $Z \times \Omega$, the trajectories of $\hat{\xi}, \hat{\rho}$ in (20) may escape from the domain $Z \times \Omega$. Thus Assumption 3.3 should be extended to a large domain to prove the error dynamic stability, which is not obvious. A natural treatment is to prevent the state and parameter estimates in $\hat{\varphi}$ from leaving the bounded domain $Z \times \Omega$, by saturating the arguments $\hat{\xi}$ and $\hat{\rho}$ in (22), which consequentially guarantees the satisfaction of Assumptions 3.2-3.3. By the aforementioned saturation definition, the Lipschitz condition (23) still holds because

$$
\|\varphi-\hat{\varphi}\| \leq L\|X-\operatorname{sat}(\hat{X})\| \leq L\|X-\hat{X}\|,
$$

where $X=(\xi, \rho)$ and $\hat{X}=(\hat{\xi}, \hat{\rho})$ for any $X \in Z \times \Omega$ and $\hat{X} \in$ $\mathbb{R}^{3}$. Similar saturation technique has been applied to obtain the semi-globally stable estimation error dynamics in [43], [53].

Assumption [47, Assum. (A4')], restricted to system (18), is written as follows

Assumption 3.11: The input $u$ is such that for any trajectory of system (20), $\Upsilon(t)$ are persistently exciting i.e., , there exist $\delta_{1}, \delta_{2}, T>0$, for any $t \geq 0$, the following inequalities hold

$$
\delta_{1} I_{2} \leq \int_{t}^{t+T} \Upsilon^{T}(t) \Upsilon(t) \mathrm{d} \tau \leq \delta_{2} I_{2},
$$

where $I_{2}$ is the $2 \times 2$ identity matrix.

We have the following result on the adaptive observer design for system (18). Proof of Proposition 3.12 is omitted due to its similarity to that of [47, Thm. 4.2].

Proposition 3.12: Provided that $u, \dot{u}, y \in \mathscr{D}$, and the Lipschitz condition (19) and Assumption 3.11 hold, (20) with $\hat{\varphi}$ given by (21) is an adaptive observer of system (16), where $\theta$ is a sufficiently large positive constant.

Next we verify that Assumption 3.11 may still hold for (20) even if Assumption [47, Assum. (A3')] is not satisfied. The $\Upsilon$-dynamics is excited by the following input

$$
\frac{\partial \hat{\varphi}}{\partial \hat{\rho}}=\left[\begin{array}{c}
\beta_{1} \exp \left(\frac{\beta_{3}+\operatorname{sat}\left(\hat{\gamma}_{1}\right) u-y}{\beta_{1}}\right) u \\
\operatorname{sat}(\hat{\alpha}) \exp \left(\frac{\beta_{3}+\operatorname{sat}\left(\hat{\gamma}_{1}\right) u-y}{\beta_{1}}\right) u^{2}+\dot{u}
\end{array}\right] .
$$

Given $\theta$ a sufficiently large positive constant, we have the approximation $\Upsilon \approx \frac{1}{\theta} \frac{\partial \hat{\varphi}}{\partial \hat{\rho}}$. Hence, (24) is approximated by

$$
\begin{aligned}
\delta_{1} \theta^{2} I_{2} & \leq \int_{t}^{t+T}\left[\begin{array}{ll}
\left(\frac{\partial \hat{\varphi}}{\partial \hat{\alpha}}\right)^{2} & \frac{\partial \hat{\varphi}}{\partial \hat{\alpha}} \frac{\partial \hat{\varphi}}{\partial \hat{\gamma}_{1}} \\
\frac{\partial \hat{\varphi}}{\partial \hat{\alpha}} \frac{\partial \hat{\varphi}}{\partial \hat{\gamma}_{1}} & \left(\frac{\partial \hat{\varphi}}{\partial \hat{\gamma}_{1}}\right)^{2}
\end{array}\right] \mathrm{d} t \\
& =\left[\begin{array}{ll}
\int_{t}^{t+T}\left(\frac{\partial \hat{\varphi}}{\partial \hat{\varphi}}\right)^{2} \mathrm{~d} t & \int_{t}^{t+T} \frac{\partial \hat{\varphi}}{\partial \hat{\alpha}} \frac{\partial \hat{\varphi}}{\partial \hat{\gamma}_{1}} \mathrm{~d} t \\
\int_{t}^{t+T} \frac{\partial \hat{\varphi}}{\partial \hat{\alpha}} \frac{\partial \hat{\varphi}}{\partial \hat{\gamma}_{1}} \mathrm{~d} t & \int_{t}^{t+T}\left(\frac{\partial \hat{\varphi}}{\partial \hat{\gamma}_{1}}\right)^{2} \mathrm{~d} t
\end{array}\right] \leq \delta_{2} \theta^{2} I_{2}
\end{aligned}
$$

Since any two square-integrable real-valued functions $\chi_{1}$ and $\chi_{2}$ on an interval $[a, b]$ have an inner product

$$
\left\langle\chi_{1}, \chi_{2}\right\rangle=\int_{a}^{b} \chi_{1}(t) \chi_{2}(t) \mathrm{d} t,
$$


if assuming $\partial \hat{\varphi} / \partial \hat{\alpha}$ and $\partial \hat{\varphi} / \partial \hat{\gamma}_{1}$ are integrable over $[t, t+T]$ for any $t \geq 0$, we rewrite (24) as follows

$$
\delta_{1} \theta^{2} I_{2} \leq\left[\begin{array}{ll}
\left\langle\frac{\partial \hat{\varphi}}{\partial \hat{\alpha}}, \frac{\partial \hat{\varphi}}{\partial \hat{\alpha}}\right\rangle & \left\langle\frac{\partial \hat{\varphi}}{\partial \hat{\alpha}}, \frac{\partial \hat{\varphi}}{\partial \hat{\gamma}_{1}}\right\rangle \\
\left\langle\frac{\partial \hat{\varphi}}{\partial \hat{\alpha}}, \frac{\partial \hat{\varphi}}{\partial \hat{\gamma}_{1}}\right\rangle & \left\langle\frac{\partial \hat{\varphi}}{\partial \hat{\gamma}_{1}}, \frac{\partial \hat{\varphi}}{\partial \hat{\gamma}_{1}}\right\rangle
\end{array}\right] \leq \delta_{2} \theta^{2} I_{2}
$$

We can further verify that the space consisting of all integrable functions over $[t, t+T]$ for any $t \geq 0$ is a pre-Hilbert space and the inner product is a norm of the space, thus the CauchySchwarz inequality holds. Denoting

$$
\|\chi\|^{2}=\langle\chi, \chi\rangle=\int_{t}^{t+T} \chi^{2}(t) \mathrm{d} t
$$

we have

$$
\left\langle\frac{\partial \hat{\varphi}}{\partial \hat{\alpha}}, \frac{\partial \hat{\varphi}}{\partial \hat{\gamma}_{1}}\right\rangle \leq\left\|\frac{\partial \hat{\varphi}}{\partial \hat{\alpha}}\right\| \times\left\|\frac{\partial \hat{\varphi}}{\partial \hat{\gamma}_{1}}\right\| .
$$

That is to say, given $\|\partial \hat{\varphi} / \partial \hat{\alpha}\|$ and $\left\|\partial \hat{\varphi} / \partial \hat{\gamma}_{1}\right\|$ nonzero, the matrix in (25) is not positive definite if and only if $\forall t \geq 0$,

$$
\frac{\partial \hat{\varphi}(\tau)}{\partial \hat{\alpha}}=k \frac{\partial \hat{\varphi}(\tau)}{\partial \hat{\gamma}_{1}}, \quad \forall k \in R, \tau \in[t, t+T] .
$$

An intuitive interpretation of (26) is that the sensitivity functions of $\varphi$ with respect to parameters $\alpha$ and $\gamma_{1}$ are linearly independent, otherwise, $\alpha$ and $\gamma_{1}$ are not distinguishable.

Remark 3.13: The fact that [47, Assum. (A3')] does not hold for the transformed system (18) partially justifies the use of the two-stage approach for $\mathrm{SoC}$ and parameter estimation. For the one-stage approach where $\beta_{i}$ are unknown parameters as well, [47, Assum. (A3')] and the PEC [47, Assum. (A4')] are much more difficult to satisfy.

A similar result to Proposition 3.12, based on a different definition of $\hat{\varphi}$ and the Lipschitz condition, is given as follows.

Proposition 3.14: Provided that $u, \dot{u}, y \in \mathscr{D}$, and the Lipschitz condition (23) and Assumption 3.11 hold, (20) with $\hat{\varphi}$ given by (22) is an adaptive observer of system (16), where $\theta$ is a sufficiently large positive constant.

\section{Alternative Adaptive Observer}

In the observer (20), the priori knowledge about the bounds of the state and parameters is used to saturate the estimates in $\hat{\varphi}$ such that the error dynamic stability can be established. The observer state $\hat{\xi}$ and $\hat{\rho}$ however can unnecessarily leave the domain $Z \times \Omega$, i.e., the observer (20) does not fully exploit the priori knowledge of the state and parameter bounds. We may improve this by considering the following alternative adaptive system

$$
\begin{aligned}
& \dot{\hat{\xi}}=\theta(y-\hat{y})+\hat{\varphi}+\theta \Upsilon \operatorname{Proj}(\kappa, \hat{\rho}), \quad \hat{\xi}(0)=\xi_{0} \in Z, \\
& \dot{\Upsilon}=-\theta \Upsilon+\frac{\partial \hat{\varphi}}{\partial \hat{\rho}}, \quad \Upsilon(0)=\Upsilon_{0} \in \mathbb{R}^{2}, \\
& \dot{\hat{\rho}}=\operatorname{Proj}(\kappa, \hat{\rho}), \quad \hat{\rho}(0)=\hat{\rho}_{0} \in \Omega, \\
& \dot{P}=-\theta P \Upsilon^{T} \Upsilon P+\theta P, \quad P(0)=P_{0} \in \mathbb{R}^{2 \times 2},
\end{aligned}
$$

where $\quad \kappa=\left(\kappa_{1}, \kappa_{2}\right)^{T}=\theta P \Upsilon^{T}(y-\hat{y}), \quad \operatorname{Proj}(\kappa, \hat{\rho})=$ $\left(\operatorname{Proj}\left(\kappa_{1}, \hat{\alpha}\right), \operatorname{Proj}\left(\kappa_{1}, \hat{\gamma}_{1}\right)\right)^{T}$, and $\hat{\varphi}$ is given by $(21)$. The projection operator $\operatorname{Proj}(\cdot, \cdot)$ is defined as follows

$$
\begin{aligned}
& \operatorname{Proj}\left(\kappa_{1}, \hat{\alpha}\right)= \begin{cases}0, & \text { if } \kappa_{1} \leq 0 \text { and }(\hat{\alpha}-\underline{\alpha}) \leq 0, \\
0, & \text { if } \kappa_{1} \geq 0 \text { and }(\hat{\alpha}-\bar{\alpha}) \geq 0, \\
\kappa_{1}, & \text { otherwise, }\end{cases} \\
& \operatorname{Proj}\left(\kappa_{2}, \hat{\gamma}_{1}\right)= \begin{cases}0, & \text { if } \kappa_{2} \leq 0 \text { and }\left(\hat{\gamma}_{1}-\underline{\gamma}\right) \leq 0, \\
0, & \text { if } \kappa_{2} \geq 0 \text { and }\left(\hat{\gamma}_{1}-\bar{\gamma}\right) \geq 0, \\
\kappa_{2}, & \text { otherwise, }\end{cases}
\end{aligned}
$$

where $\gamma, \bar{\gamma}$ are lower and upper bounds of $\gamma_{1}$. The projection operator can be chosen differently, e.g. [54, Eqn. (A.25)]. We have the following result about the system (27).

Proposition 3.15: Provided that $u, \dot{u}, y \in \mathscr{D}$, and the Lipschitz condition (23) and Assumption 3.11 hold, the system (27) with $\hat{\varphi}$ given by (21) is an adaptive observer of the system (16), where $\theta$ is a sufficiently large positive constant.

A detailed proof of Proposition 3.15 is omitted, since it is can be readily established by considering the proof of Proposition 3.12 and the following fact:

According to the proof of [47, Thm. 4.2], there exists a time-varying quadratic Lyapunov function candidate $V(t, \tilde{x})$ such that its time derivative along the the error dynamics corresponding to observer (20), denoted by $\dot{V}_{(20)}$, satisfies

$$
\dot{V}_{(20)}=\frac{\partial V}{\partial t}+\frac{\partial V}{\partial \tilde{x}} \tilde{f}_{(20)} \leq-k_{4} V,
$$

where $\tilde{x}$ is the estimation error, $\tilde{f}_{(20)}$ is the estimation error dynamics corresponding to (20), and $k_{4}>0$. Next we show that the time derivative of $V$ along the error dynamics corresponding to observer (27), denoted by $\dot{V}_{(27)}$, is upper-bounded by $\dot{V}_{(20)}$, i.e., that satisfies the following inequality

$$
\dot{V}_{(27)} \leq \dot{V}_{(20)} \leq-k_{4} V
$$

Essentially, we need to show

$$
\frac{\partial V}{\partial \tilde{x}} \tilde{f}_{(27)} \leq \frac{\partial V}{\partial \tilde{x}} \tilde{f}_{(20)},
$$

where $\tilde{f}_{(27)}$ is the estimation error dynamics corresponding to (27). Since $V$ is a time-varying and quadratic function of estimation errors, we take a Lyapunov function $V_{\alpha}=0.5(\alpha-$ $\hat{\alpha})^{2}$ as an example to illustrate the proof. We have the time derivative of $V_{\alpha}$ along (27)

$\dot{V}_{\alpha}=k_{1}(\alpha-\hat{\alpha}) \operatorname{Proj}\left(\kappa_{1}, \hat{\alpha}\right)=\left\{\begin{array}{l}0, \quad \text { if } \kappa_{1} \leq 0 \text { and }(\hat{\alpha}-\underline{\alpha}) \leq 0, \\ 0, \quad \text { if } \kappa_{1} \geq 0 \text { and }(\hat{\alpha}-\underline{\alpha}) \leq 0, \\ k_{1}(\alpha-\hat{\alpha}) \kappa_{1}, \quad \text { otherwise. }\end{array}\right.$

With the same Lyapunov function, its time derivative along (20) is

$$
\dot{\bar{V}}_{\alpha}=k_{1}(\alpha-\hat{\alpha}) \kappa_{1}=\left\{\begin{array}{l}
\geq 0, \quad \text { if } \kappa_{1} \leq 0 \text { and }(\hat{\alpha}-\underline{\alpha}) \leq 0, \\
\geq 0, \quad \text { if } \kappa_{1} \geq 0 \text { and }(\hat{\alpha}-\underline{\alpha}) \leq 0, \\
k_{1}(\alpha-\hat{\alpha}) \kappa_{1}, \quad \text { otherwise. }
\end{array}\right.
$$

We know

$$
\dot{V}_{\alpha} \leq \dot{\bar{V}}_{\alpha} .
$$

This fact holds for the estimation errors $\xi-\hat{\xi}$ and $\gamma_{1}-\hat{\gamma}_{1}$. We therefore prove (30). We therefore conclude that the adaptive observer (27) also yields exponentially stable error dynamics. 
Remark 3.16: Assumption 3.11 imposes different constraints on the systems (20) and (27), respectively. That is to say, the satisfaction of Assumption 3.11 for the system (20) does not necessarily concur with its satisfaction for the system (27).

Similar to Proposition 3.15, we have the following statement.

Proposition 3.17: Provided that $u, \dot{u}, y \in \mathscr{D}$, and the Lipschitz condition (23) and Assumption 3.11 hold, the system (27) with $\hat{\varphi}$ given by (22) is an adaptive observer of the system (16), where $\theta$ is a sufficiently large positive constant.

Remark 3.18: Another form of adaptive observer can be taken by applying projection operator to the right hand side of the dynamic equation of $\hat{\xi}$, i.e., the first equation of (27) is replaced with the following equation

$$
\dot{\hat{\xi}}=\operatorname{Proj}(\psi, \hat{\xi}),
$$

where $\psi=\theta(y-\hat{y})+\hat{\varphi}+\theta \Upsilon \operatorname{Proj}(\kappa, \hat{\rho})$. Error dynamics stability can be established by using the same technique as in the sketched proof of Proposition 3.15.

\section{E. Robustness and Applicability}

Given Assumption 3.11, both of adaptive observers (20) and (27) yield error dynamics of the state and parameter estimation which are exponentially convergent. We analyze the robustness of the adaptive observer (20) with respect to the process model uncertainty and the measurement noise. The adaptive observer (27) enjoys similar robustness property as (20) thus its analysis is omitted.

Robustness analysis is performed based on the input-statestability (ISS) [55, Def. 4.7]. To facilitate the analysis, we introduce the notation of estimation errors: $\tilde{X}=X-\hat{X}$. Given the system (18) and the adaptive observer (20), it is not difficult to derive that the error dynamics can be written as follows

$$
\dot{\tilde{X}}=\tilde{f}(y, u, \dot{u}, \operatorname{sat}(\hat{\xi}), \operatorname{sat}(\hat{\rho}), \theta, \Upsilon, P, \tilde{X}),
$$

where $y, u, \dot{u}, \operatorname{sat}(\hat{\xi}), \operatorname{sat}(\hat{\rho}), \theta, \Upsilon, P$ are bounded. Considering $\tilde{f}$ is continuously differentiable and $\varphi$ is Lipschitz over $\mathscr{D}$, we know that $\partial \tilde{f} / \partial \tilde{X}$ is bounded over $Z \times \Omega$, uniformly in $y, u, \dot{u}, \operatorname{sat}(\hat{\xi}), \operatorname{sat}(\hat{\rho}), \theta, \Upsilon, P$. Also since the zero solution of the $\tilde{X}$-dynamics is exponentially stable, [55, Thm. 4.14] applies, i.e., there exists a function $V(t, \tilde{X})$ satisfying the inequalities:

$$
\begin{aligned}
& c_{1}\|\tilde{X}\|^{2} \leq V \leq c_{2}\|\tilde{X}\|^{2}, \\
& \frac{\partial V}{\partial t}+\frac{\partial V}{\partial \tilde{X}} \tilde{f} \leq-c_{3}\|\tilde{X}\|^{2}, \\
& \left\|\frac{\partial V}{\partial \tilde{X}}\right\| \leq c_{4}\|\tilde{X}\|,
\end{aligned}
$$

where $c_{i}, 1 \leq i \leq 4$ are positive constants. Now assume instead of (16), the true battery model is given by

$$
\begin{aligned}
& \dot{x}=\alpha u+d(t), \\
& y=\beta_{1} \log \left(x+\beta_{2}\right)+\beta_{3}+\gamma_{1} u+n(t),
\end{aligned}
$$

where $d(t)$ is bounded and represents the unmodeled process dynamics, and $n(t)$ is continuously differentiable and represents the measurement noise. Under the new coordinates defined by (17), the system (33) is rewritten as follows

$$
\begin{aligned}
\dot{\xi} & =\varphi(y-n(t), u, \dot{u}, \rho)+d(t), \\
y & =\xi+n(t),
\end{aligned}
$$

With adaptive observer (20), the resultant error dynamics are $\dot{\tilde{X}}=\tilde{f}_{u}(y-n(t), u, \dot{u}, \operatorname{sat}(\hat{\xi}), \operatorname{sat}(\hat{\rho}), \theta, \Upsilon, P, \tilde{X})+E n(t)+F d(t)$, where

$$
E=\left[\begin{array}{c}
\theta+\theta^{2} \Upsilon P \Upsilon^{T} \\
\theta P \Upsilon^{T}
\end{array}\right] \in \mathbb{R}^{3}, \quad F=\left[\begin{array}{l}
1 \\
0 \\
0
\end{array}\right] .
$$

Assuming inequalities (32) still hold with $\tilde{f}$ replaced by $\tilde{f}_{u}$, we have

$$
\begin{aligned}
\dot{V} & =\frac{\partial V}{\partial t}+\frac{\partial V}{\partial \tilde{X}}\left(\tilde{f}_{u}+E n(t)+F d(t)\right) \\
& \leq-c_{3}\|\tilde{X}\|^{2}+c_{4}\|\tilde{X}\| \cdot(\|E n(t)+F d(t)\|), \\
& \leq-c_{3}\|\tilde{X}\|^{2}+c_{4}\|\tilde{X}\| \cdot\left(\|E\| \cdot\|n(t)\|_{\infty}+\|F\| \cdot\|d(t)\|_{\infty}\right) \\
& \leq-c_{3}\|\tilde{X}\|^{2}+c_{4} c_{5}(\theta)\|n(t)\|_{\infty} \cdot\|\tilde{X}\|+c_{4}\|d(t)\|_{\infty} \cdot\|\tilde{X}\|,
\end{aligned}
$$

where $c_{5}$ is positive, and $\|n(t)\|_{\infty},\|d(t)\|_{\infty}$ are the infinity norm of signals $n(t)$ and $d(t)$, respectively. From the expression of $\dot{V}$, we have the following conclusions

1) The error dynamics are ISS with respect to unmodeled process dynamics and the measurement noise, i.e., given bounded unmodeled process dynamics $d(t)$ and the measurement noise $n(t)$, the estimation error is bounded.

2) Since $c_{5}$ is a function of $\theta$, the term $c_{4} c_{5}(\theta)\|n(t)\|_{\infty}$. $\|\tilde{X}\|$, due to the measurement noise, could be quite large. On the other hand, the term $c_{4}\|d(t)\|_{\infty} \cdot\|\tilde{X}\|$, due to the unmodeled process dynamics, is independent from $\theta$. Hence, the adaptive observer (20) is more robust to the unmodeled process dynamics than to the measurement noise. This is not surprising due to the high gain essence of the adaptive observer (20).

3) The adaptive observer (27) is more robust with respect to the measurement noise than (20) because the adaptive law in (20), i.e., the $\hat{\rho}$-dynamics, do not use the projection operator, thus the term $\theta^{2} \Upsilon P \Upsilon^{T}$ always appears in the $\tilde{\xi}$-dynamics. Instead, for the adaptive observer (27), during the period of $\operatorname{Proj}(\kappa, \hat{\rho})=0$, the term $\theta^{2} \Upsilon P \Upsilon^{T}$, representing the effect of the measurement noise, is absent from the $\tilde{\xi}$-dynamics.

4) There exists a tradeoff between the convergence rate of the error dynamics and the robustness with respect to the measurement noise. Given the PEC satisfied for some $\delta_{1}, \delta_{2}$ and a sufficiently large $\theta$, a larger $\theta$, from theoretical perspective, leads to faster convergence at the expense of more sensitivity to the measurement noise. Making the gain $\theta$ time varying might help to improve the transience of the error dynamics, for instance, adjusting the gain $\theta$ according to the amplitude of the estimation error $y-\hat{y}$.

Both of adaptive observers (20) and (27) rely on the transformed system (18), which implies continuous differentiability 
of the transformed state $\xi$. Equivalently both the adaptive observers (20) and (27) assume the continuous differentiability of the terminal voltage. This assumption may not be valid in certain scenario. For instance, the input current $u$ may not be differentiable or even continuous, so does the terminal voltage.

The system's failure to satisfy the differentiability prerequisite does not mean the adaptive observers (20) and (27) are not applicable to that system. The adaptive observer (20) or (27) is essentially an filter which tries to track the terminal voltage. The discontinuities in the terminal voltage can be viewed as various step inputs at different time instants. Since the filter has a limited bandwidth controlled by the gain $\theta$, it cannot track the step input perfectly and a transient response of the estimated terminal voltage is resulted. Since the terminal voltage estimation is clearly not accurate, thus the SoC and parameter estimates deviate from the true values during the transience. The applicability of the proposed adaptive observers can be summarized as follows:

1) The proposed approach is applicable for the cases where the terminal voltage is continuously differentiable. This scenario includes applications where the charge and discharge processes are completely separate. For instance portable electronic devices. For these applications, the proposed approach leads to an adaptive observer which provides reliable estimation of both the SoC for control and parameters for battery diagnosis.

2) Similarly, the proposed approach is applicable to cases where charge and discharge periods are much longer than the transient period due to jumps in the terminal voltage. Note that the transient period can be made arbitrarily small, theoretically, by choosing a sufficiently large observer gain $\theta$. In practice this is not possible because of the design tradeoff between the gain $\theta$ and the noise level of the measurements, and the constraint on $\theta$ to satisfy the PEC. Measurements with higher resolution and quality allow a larger $\theta$ which leads to a shorter transient time. A variable gain strategy, for instance based on the amplitude of the estimation error of the terminal voltage, however could relax the fundamental limitation.

3) The proposed approach can complement other auxiliary estimators, which are insensitive to jumps in the input current, to produce a good estimate. Such an auxiliary estimator can be based on Coulomb counting or Kalman Filtering.

It is worth pointing out that the proposed approach is generic and not limited to the specific model (12). Generalization of the proposed approach to other models might also be possible, for instance equivalent circuit models with or without the hysteresis voltage as a state variable.

\section{F. A Switched Adaptive Observer}

This section includes a brief illustration of the adaptive SoC estimation based on the switched battery model (11). Similar to the procedure in Section III-C, we introduce state transformations $\xi_{1}=h(x, \beta)+g(u, \gamma)$ and $\xi_{2}=h(x, \bar{\beta})+g(u, \gamma)$ corresponding to discharge and charge modes, respectively, and have the transformed system given by

$$
\begin{aligned}
& \Sigma_{d}:\left\{\begin{array}{l}
\dot{\xi}_{1}=\varphi_{1}(\cdot, \beta), \quad t \geq t_{i}, \\
y=\xi_{1},
\end{array}\right. \\
& \Sigma_{c}:\left\{\begin{array}{l}
\dot{\xi}_{2}=\varphi_{2}(\cdot, \bar{\beta}), \quad t \geq t_{j}, \\
y=\xi_{2},
\end{array}\right.
\end{aligned}
$$

where $t_{i}, t_{j}$ are the time instants when the sign of $u$ changes. To enforce the continuity of the SoC, we allow jumps of the state $\xi_{1}$ or $\xi_{2}$ at switch times $t_{i}$ and $t_{j}$, i.e., the following switch conditions hold

$$
\begin{aligned}
& \xi_{1}\left(t_{i}^{+}\right)=h\left(x\left(t_{i}^{-}\right), \bar{\beta}\right)+g\left(u\left(t_{i}^{+}\right), \gamma\left(t_{i}^{+}\right)\right), \\
& \xi_{2}\left(t_{j}^{+}\right)=h\left(x\left(t_{j}^{-}\right), \beta\right)+g\left(u\left(t_{j}^{+}\right), \gamma\left(t_{j}^{+}\right)\right) .
\end{aligned}
$$

The system (35) is impulsive and the observer design for these systems is out of main focus of this paper. Given system (35)(36), one can apply the proposed approach to $\Sigma_{d}$ and $\Sigma_{c}$ respectively. If $u$ switches sign slowly enough, the stability results in Propositions 3.12-3.15 can be similarly established for the adaptive system consisting of two adaptive observers which are designed on the basis of $\Sigma_{d}$ and $\Sigma_{c}$, respectively.

\section{EXAMPLES}

\section{A. Simulation}

Consider the battery model (10) and assume that there is no mismatch between the model and the battery dynamics. The model parameters are given as follows: $\alpha=4.7496 \times 10^{-5}$, $\beta_{1}=1.0480, \beta_{2}=0.2208, \beta_{3}=3.9998, \gamma_{1}=-5 \times 10^{-3}$. Here, the values of $\alpha$ and $\gamma_{1}$ are reckoned according to [24], [25], [56] and may have little applicability to a specific battery. The values of $\beta_{i}$ 's are determined by fitting the SoC-OCV data of the battery from experiments. The input to the model is a sinusoid wave $u=10 \sin (10 t)$. We take $\theta=20$ and the following initial conditions (ICs)

$$
\begin{aligned}
& \xi(0)=0.5 ; \quad \hat{\xi}(0)=0 ; \\
& \Upsilon(0)=(0,0), \quad \hat{\rho}(0)=(0,0)^{T}, \quad P(0)=I_{2} .
\end{aligned}
$$

Simulation results are given in Figures 3-5, which show that adaptive observer (20) can provide convergent estimation of the transformed system state and parameters. This further implies the state of the original system (16), or the SoC, can also be estimated exponentially. We also verify that the adaptive observer provides convergent estimation of the SoC and parameters over a fairly large domain. Details are omitted due to space limitation. Interested readers are referred to [22] for details.

\section{B. Experiment}

The experimental validation of the proposed approach is given as follows. For the case where the adaptive SoC estimation is performed based on the adaptive observer (20) and the model (10), we use both charge and discharge curves to identify parameters $\beta$ in $h(x, \beta)$. For the case where the adaptive SoC estimation is performed based on adaptive observer (27) and the switched model (11), we use the charge and discharge curves to identify parameters $\beta$ and $\bar{\beta}$, respectively. Both the 

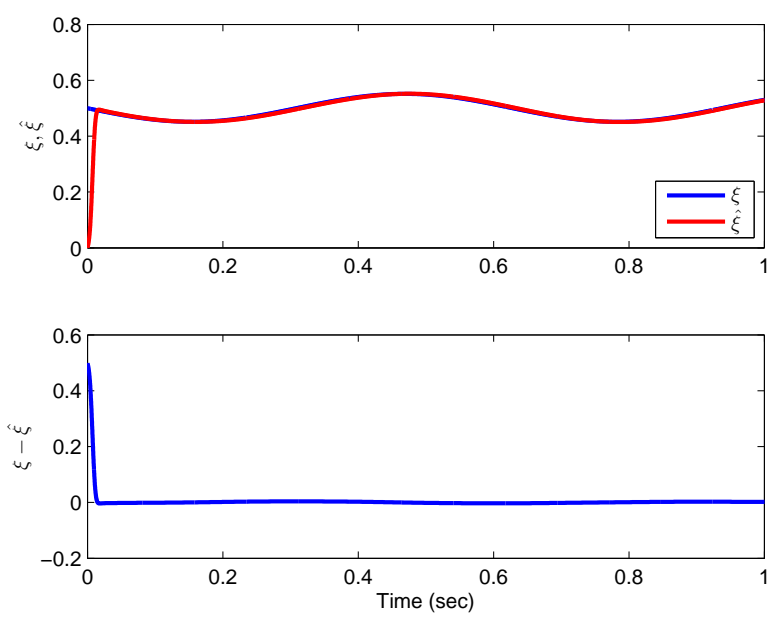

Fig. 3. The transformed system state $\xi$ and its estimation $\hat{\xi}$
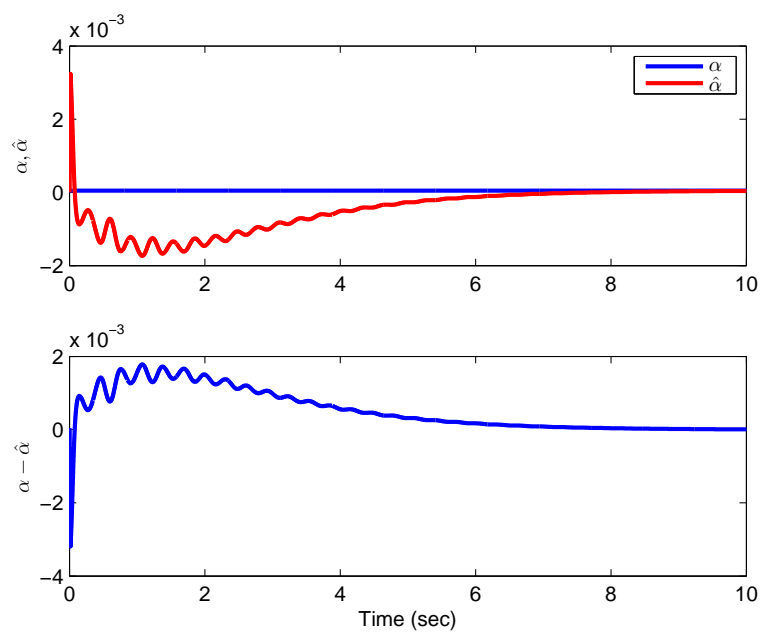

Fig. 4. Parameter $\alpha$ and its estimation $\hat{\alpha}$
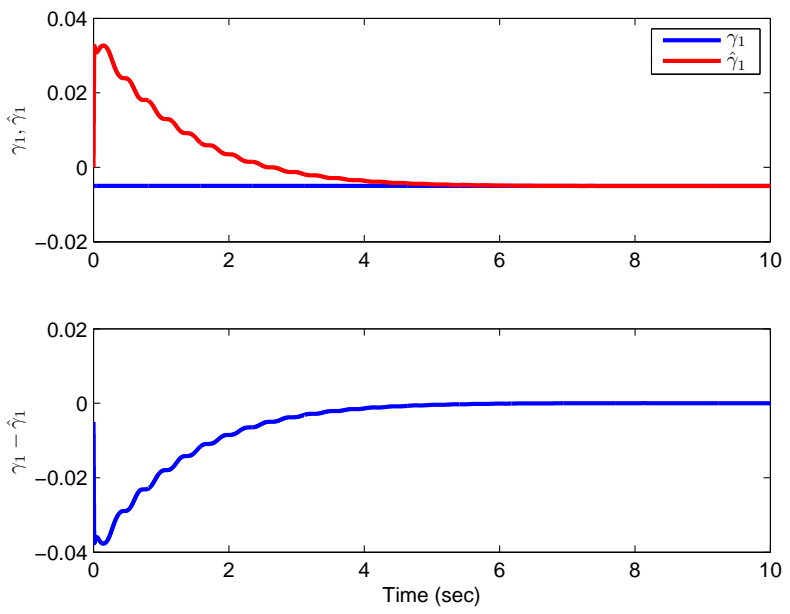

Fig. 5. Parameter $\gamma_{1}$ and its estimation $\hat{\gamma}_{1}$

charge and discharge curves depict the relationship between the $\mathrm{SoC}$ and the terminal voltage of the battery in charge and discharge modes, respectively, where the $\mathrm{SoC}$ is obtained by coulomb counting. Instead of having the battery rest for a long time to measure the OCV, we measure the terminal voltage continuously as the battery is in charge or discharge modes. Particularly, the charge curve is obtained by applying a small charge current to the battery and the discharge curve is obtained by applying a small discharge current to the battery.

The rest experiment to validate the SoC estimation algorithm is conventional. That is: the battery is subject to a charge and discharge current; both the current and the terminal voltage are measured. The input current profile is shown in Figure 6 . The battery has a nominal capacity 4.903Ah. The

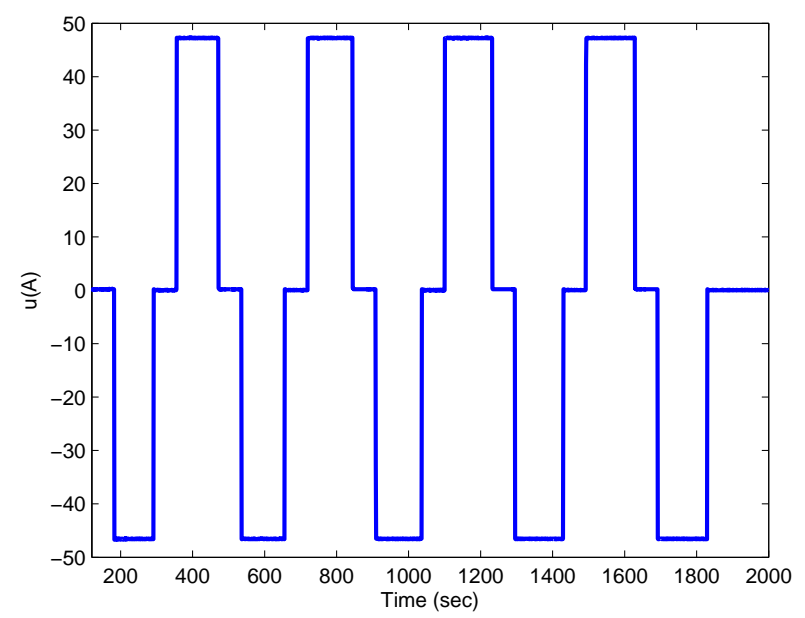

Fig. 6. The input current of the battery

input current and terminal voltage are sampled every second. Thus the nominal value of the parameter $\alpha$ is $5.63421 e \times 10^{-5}$. The ICs of adaptive observers are taken as follows

$$
\begin{aligned}
& \hat{\xi}(0)=3, \\
& \Upsilon(0)=(0,0), \quad \hat{\rho}(0)=(0,0)^{T}, \quad P(0)=I_{2} .
\end{aligned}
$$

The gain tuning parameter $\theta$ is taken as 0.08 for adaptive observer (20) and 0.025 for adaptive observer (27). The validation results are shown in Figures 6-10, where red solid lines correspond to results of adaptive observer (20) and the black line corresponds to adaptive observer (27). The transformed system state, the state estimate, and their error are shown in Figure 7. Overall the state estimate $\hat{\xi}$ tracks the true state $\xi$ except spikes at time instants when the input current jumps. It is worth pointing out that the estimation error approaches zero over time interval between two adjacent current jumps.

The estimation of the parameter $\alpha$ is shown in Figure 8, where the blue solid line represents the nominal value of $\alpha$. Consistent with the trajectory of the state estimation error $\xi-\hat{\xi}$, the trajectory of $\hat{\alpha}$ has spikes when the current changes directions. The appearance of spikes in $\hat{\alpha}$ is understood because the proposed adaptive observer updates its estimates according to the spiky output error $\xi-\hat{\xi}$. The spikes in the trajectory of $\xi-\hat{\xi}$, interpreted as step inputs into the adaptive observer, necessarily induce spikes in $\hat{\alpha}$. Although the 

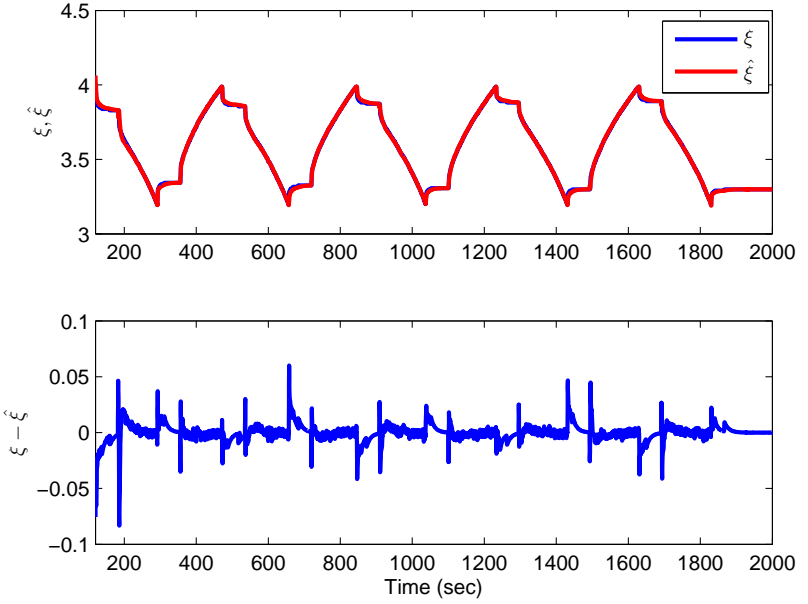

Fig. 7. The transformed system state $\xi$ and its estimation $\hat{\xi}$

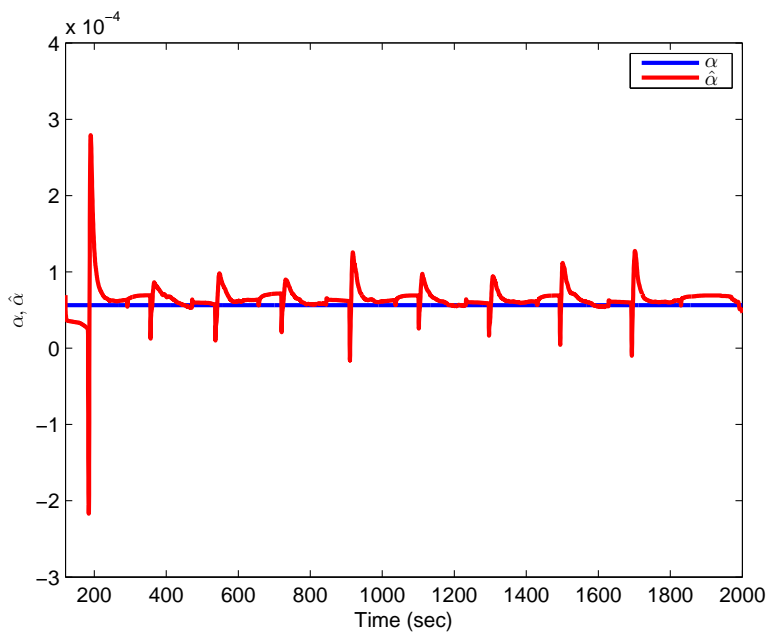

Fig. 8. Estimate of the parameter $\alpha$

amplitude of $\hat{\alpha}$ around spikes may be several times larger than the nominal value of $\alpha$, the trajectory of $\hat{\alpha}$ seems convergent to some extent during the interval between jumps, and is still useful. This is because the trajectory of $\hat{\alpha}$ stays in a small neighborhood of the nominal value at most of time, and functions of $\hat{\alpha}$, e.g. the mean value, might indicate the State of Health in the long run. The estimation of the parameter $\gamma_{1}$ is given in Figure 9. Again the parameter estimate $\hat{\gamma}_{1}$ has spikes. It should be realized that the estimate $\hat{\gamma}_{1}$ is not so valuable during periods with zero input current, because the effect of $\gamma_{1}$ is literally ignored by the model. If we ignore those zero current intervals, it is not difficult to see that during the intervals between two adjacent current jumps, the $\hat{\gamma}_{1}$ trajectory approaches to some value between $2.5 \mathrm{~m}-\mathrm{Ohm}$ and $3 \mathrm{~m}-\mathrm{Ohm}$. Overall, the estimate $\hat{\gamma}_{1}$ is quite reasonable in the sense that the average of the estimate trajectory has a small variation.

The estimates of the SoC using Coulomb counting and the proposed approach are presented in Figures 10, where the red solid line is the SoC estimate produced by adaptive observer (20) designed based on (18), and the black solid line is the SoC

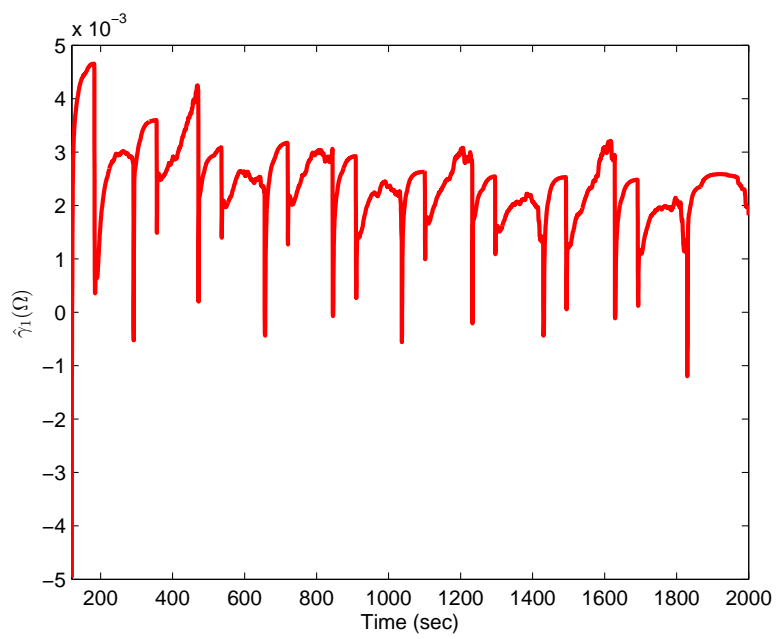

Fig. 9. Estimate of the parameter $\gamma_{1}$

estimate produced by adaptive observer (27) designed based on (35). The implemented adaptive observer (27) however still enforces the continuity of the terminal voltage instead of the SoC. This is to examine how the introduction of the hysteresis in the OCV-SoC curve can improve the accuracy of the SoC estimation. Consistent with trajectories of the state and parameter estimates, the estimated SoC trajectory also has spikes which at the worst case could shoot up to $10 \%$ for the case ignoring hysteresis. A number of factors contribute to the appearance of spikes. First factor is the presence of

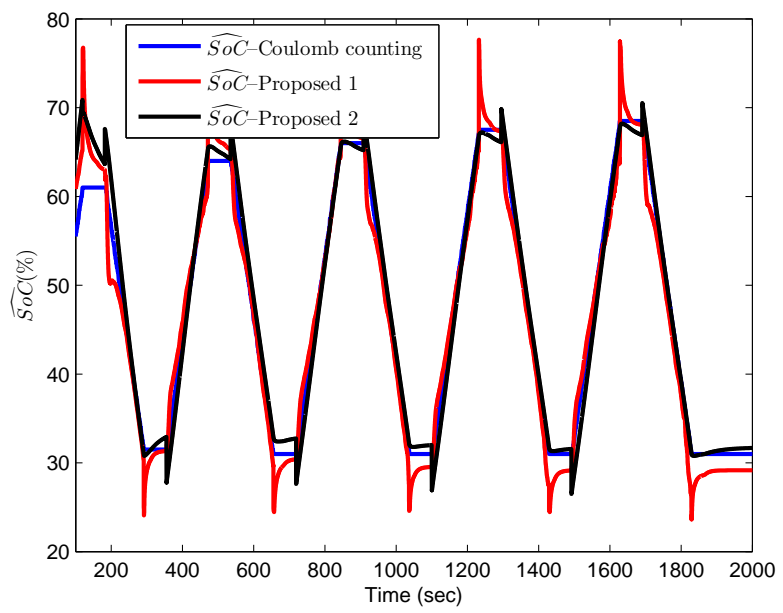

Fig. 10. SoC estimates $\widehat{S o C}$ using various methods

the discontinuity of the input current. We illustrate this by considering the scenario where the input current switches from non-zero to zero at time $t_{1}$. At time $t_{1}^{-}$, the terminal voltage is $y\left(t_{1}^{-}\right)=O C V\left(t_{1}^{-}\right)+\gamma_{1} u\left(t_{1}^{-}\right)$; at the time instant $t_{1}^{+}$, the terminal voltage is $y\left(t_{1}^{+}\right)=O C V\left(t_{1}^{+}\right)$. Since the proposed approach assumes the continuity of the terminal voltage, i.e.,

$$
y\left(t_{1}^{-}\right)=O C V\left(t_{1}^{-}\right)+\gamma_{1} u\left(t_{1}^{-}\right)=y\left(t_{1}^{+}\right)=O C V\left(t_{1}^{+}\right),
$$

the $\mathrm{OCV}$, consequently the $\mathrm{SoC}$, at $t_{1}^{+}$has to be discontinuous. 
In the experiment, the amplitude of the current is around 50A. Given $\gamma_{1}=2.5 \mathrm{~m}-\mathrm{Ohm}$, the jump of OCV from $t_{1}^{-}$to $t_{1}^{+}$will be \pm 0.125 Volts. According to the average SoC-OCV curve, variations of the $\mathrm{OCV}$ with such an amplitude corresponds to around $5-10 \%$ variation of the SoC. In a realistic scenario, it is unlikely that the input current is discontinuous thus the proposed algorithm might be still applicable. One solution to this issue is to modify the proposed algorithm to be free of time derivative.

The second factor is the model mismatch, i.e., the model (10) fails to capture the RCE, the RE, and the HE. The HE on the SoC estimation has been alleviated by applying the proposed approach to the switched battery model (11). As shown in Figure 10, the SoC estimation error corresponding to the switched model is within $4.5 \%$. Further improvement of the $\mathrm{SoC}$ estimation accuracy by enforcing the continuity of the $\mathrm{SoC}$ is possible.

\section{CONCLUSion AND Future Work}

This paper considered adaptive State of Charge (SoC) and parameter estimation of Lithium-ion batteries. A wellestablished nonlinear geometric observer approach was applied to simplified and nonlinear battery models for estimating the SoC and parameters. The resultant error dynamics of state and parameter estimation are exponentially convergent under the model matching condition. The proposed adaptive observers were shown robust to process uncertainties. Robustness analysis also indicated the design tradeoff between the convergence rate of the error dynamics and robustness to the measurement noise. Simulation and experiments were carried out to validate the effectiveness and main advantages of the proposed approach: capability to provide relatively reliable estimates of the $\mathrm{SoC}$ and parameters. Experiments also revealed that the proposed approach may not work well if the input current has many discontinuous points. Error analysis was presented to explain experimental results. Future work will focus on the modification of the proposed algorithm to be free of input derivative, and its generalization to other models.

\section{REFERENCES}

[1] Y. Nishi, "Lithium ion secondary batteries; past 10 years and the future," J. of Power Sources, vol. 100, no. 1-2, pp. 101-106, 2001.

[2] N. Chaturvedi, R. Klein, J. Christensen, J. Ahmed, and A. Kojic, "Algorithms for advanced battery-management systems," IEEE Contr. Sys. Mag., vol. 30, no. 3, pp. 49-68, 2010.

[3] V. Pop, H. J. Bergveld, P. H. L. Notten, and P. P. L. Regtien, "State-ofthe-art of battery state-of-charge determination," Measurement Science and Technology, vol. 16, no. 12, pp. R93-R110, 2005.

[4] J. Chiasson and B. Vairamohan, "Estimating the state of charge of a battery," IEEE Trans. Contr. Syst. Technol., vol. 13, no. 3, pp. 465-470, 2006.

[5] G. L. Plett, "Extended Kalman filtering for battery management systems of LiPB-based HEV battery packs: Part 3. state and parameter estimation," J. of Power Sources, vol. 134, no. 2, pp. 277-292, 2004.

[6] _ - "Sigma-point Kalman filtering for battery management systems of LiPB-based HEV battery packs: Part 2: Simultaneous state and parameter estimation," J. of Power Sources, vol. 161, no. 2, pp. 1369 1384, 2006.

[7] I.-S. Kim, "The novel state of charge estimation method for lithium battery using sliding mode observer," J. of Power Sources, vol. 163, no. 1 , pp. 584-590, 2006.
[8] M. Verbrugge and E. Tate, "Adaptive state of charge algorithm for nickel metal hydride batteries including hysteresis phenomena," J. of Power Sources, vol. 126, no. 1-2, pp. 236-249, 2004.

[9] Y. Hu and S. Yurkovich, "Battery cell state-of-charge estimation using linear parameter varying system techniques," J. of Power Sources, vol. 198, pp. 338-350, 2012.

[10] M. Doyle, T. F. Fuller, and J. Newman, "Modeling of galvanostatic charge and discharge of the lithium/polymer/insertion cell," J. Electrochem. Soc., vol. 140, no. 6, pp. 1526-1533, 1993.

[11] T. F. Fuller, M. Doyle, and J. Newman, "Simulation and optimization of the dual lithium ion insertion cell," J. Electrochem. Soc., vol. 141, no. 1, pp. 1-10, 1994.

[12] S. Atlung, K. West, and T. Jacobsen, "Dynamic aspects of solid solution cathodes for electrochemical power sources," J. Electrochem. Soc., vol. 126, no. 8, pp. 1311-1321, 1979.

[13] S. K. Rahimian, S. Rayman, and R. E. White, "Extension of physicsbased single particle model for higher charge-discharge rates," $J$. of Power Sources, vol. 224, pp. 180-194, 2013.

[14] K. A. Smith, C. D. Rahn, and C.-Y. Wang, "Model-based electrochemical estimation of lithium-ion batteries," in Proceedings of IEEE International Conference on Control Applications, 2008, pp. 714-719.

[15] D. Domenico, G. Di Fiengo, and A. Stefanopoulou, "Lithium-ion battery state of charge estimation with a Kalman filter based on a electrochemical model," in Proceedings of IEEE International Conference on Control Applications, 2008, pp. 702-707.

[16] S. Santhanagopalan and R. E. White, "State of charge estimation using an unscented filter for high power lithium ion cells," International Journal of Energy Research, vol. 34, no. 2, pp. 152-163, 2010.

[17] R. Klein, N. A. Chaturvedi, J. Christensen, J. Ahmed, R. Findeisen, and A. Kojic, "Electrochemical model based observer design for a lithiumion battery," IEEE Trans. Contr. Syst. Technol., vol. 21, no. 2, pp. 289301, Mar. 2013.

[18] S. J. Moura, N. A. Chaturvedi, and M. Krstic, "PDE estimation techniques for advanced battery management systems - Part I: SOC estimation," in Proc. 2012 ACC, 2012, pp. 559-565.

[19] O. Barbarisi, F. Vasca, and L. Glielmo, "State of charge Kalman filter estimator for automotive batteries," Control Eng. Pract., vol. 14, no. 3, pp. 267-275, 2006.

[20] M. McIntyre, T. Burg, D. Dawson, and B. Xian, "Adaptive state of charge (SOC) estimator for a battery," in Proc. 2006 ACC, 2006, pp. $5740-5744$.

[21] H. Fang, Y. Wang, Z. Sahinoglu, T. Wada, and S. Hara, "Adaptive robust estimation of state of charge for lithium-ion batteries," in Proc. 2013 ACC, Washington, DC, 2013, pp. 3491-3497.

[22] Y. Wang, H. Fang, Z. Sahinoglu, T. Wada, and S. Hara, "Nonlinear adaptive estimation of the state of charge for lithium-ion batteries," in Proc. 52th CDC, Florence, Italy, Dec. 2013, pp. 4405-4410.

[23] R. H. Milocco, J. E. Thomas, and B. E. Castro, "Generic dynamic model of rechargeable batteries," J. of Power Sources, vol. 246, pp. 609-620, 2014.

[24] S. Santhanagopalan, Q. Guo, P. Ramadass, and R. E. White, "Review of models for predicting the cycling performance of lithium ion batteries," J. of Power Sources, vol. 156, no. 2, pp. 620-628, 2006.

[25] M. Guo, G. Sikha, and R. E. White, "Single-particle model for a lithiumion cell: Thermal behavior," J. Electrochem. Soc., vol. 158, no. 2, pp. A122-A132, 2011.

[26] M. Coleman, C. K. Lee, C. Zhu, and W. G. Hurley, "State-of-Charge determination from EMF voltage estimation: Using impedance, terminal voltage, and current for lead-acid and lithium-ion batteries," IEEE Trans. Ind. Electr., vol. 54, no. 5, pp. 2550-2557, 2007.

[27] M. Gevers and G. Bastin, "A stable adaptive observer for a class of nonlinear second-order systems," in Analysis and Optimization of Systems, A. Bensoussan and J. L. Lion, Eds. New York: Springer, 1986, pp. 143-155.

[28] G. A. F. Seber and C. J. Wild, Nonlinear Regression. Wiley, 2003.

[29] J. Nocedal and S. J. Wright, Numerical Optimization. Springer, 2006.

[30] R. Marino and P. Tomei, Nonlinear Control Design: Geometric, Adaptive, and Robust. Hertfordshire, UK: Prentice-Hall, 1995.

[31] A. J. Krener and W. Respondek, "Nonlinear observers with linearizable error dynamics," SIAM J. Control Optim., vol. 23, no. 2, pp. 197-216, Mar. 1985.

[32] G. Besançon, "On output transformations for state linearization up to output injection," IEEE Trans. Automat. Control, vol. 44, no. 10, pp. 1975-1981, Oct. 1999.

[33] A. J. Krener and A. Isidori, "Linearization by output injection and nonlinear observers," Syst. Control Lett., vol. 3, no. 1, pp. 47-52, Jun. 1983. 
[34] W. Respondek, A. Pogromsky, and H. Nijmeijer, "Time scaling for observer design with linearizable error dynamics," Automatica, vol. 40, no. 2, pp. 277-285, Feb. 2004.

[35] M. Guay, "Observer linearization by output diffeomorphism and outputdependent time-scale transformations," in NOLCOS'01, St. Petersburg, Russia, 2001, pp. 1443-1446.

[36] Y. Wang and A. F. Lynch, "Multiple time scalings of a multi-output observer form," IEEE Trans. Automat. Control, vol. 55, no. 4, pp. 966 971, Apr. 2010.

[37] J. Back, K. T. Yu, and J. H. Seo, "Dynamic observer error linearization," Automatica, vol. 42, pp. 2195-2200, Dec. 2006.

[38] A. F. Lynch and S. A. Bortoff, "Nonlinear observers with approximately linear error dynamics: The multivariable case," IEEE Trans. Automat. Control, vol. 46, no. 6, pp. 927-932, Jun. 2001.

[39] N. Kazantzis and C. Kravaris, "Nonlinear observer design using Lyapunov's Auxilliary Theorem," in Proc. 36th CDC, San Diego, CA, 1997, pp. 4802-4807.

[40] J. Rudolph and M. Zeitz, "Block triangular nonlinear observer normal form," Syst. Control Lett., vol. 23, no. 1, pp. 1-8, Jul. 1994.

41] J. Schaffner and M. Zeitz, "Decentral nonlinear observer design using a block-triangular form," Internat. J. Systems Sci., vol. 30, no. 10, pp. 1131-1142, Oct. 1999.

[42] Y. Wang and A. Lynch, "A block triangular form for nonlinear observer design," IEEE Trans. Automat. Control, vol. 51, no. 11, pp. 1803-1808, Nov. 2006

[43] — "A block triangular observer forms for nonlinear observer design," Int. J. Control, vol. 81, no. 2, pp. 177-188, 2008.

[44] — , "Observer design using a generalized time scaled block triangular observer form," Syst. Control Lett., vol. 58, no. 5, pp. 346-352, May 2009.

[45] G. Bastin and M. R. Gevers, "Stable adaptive observers for nonlinear time-varying systems," IEEE Trans. Automat. Control, vol. 33, no. 7, pp. $650-658$, Jul. 1988.

[46] R. Marino and P. Tomei, "Global adaptive observers for nonlinear systems via filtered transformations," IEEE Trans. Automat. Control, vol. 37, no. 8, pp. 1239-1245, Aug. 1992.

[47] M. Farza, M. M'Saad, T. Maatoug, and M. Kamoun, "Adaptive observers for nonlinearly parameterized class of nonlinear systems," Automatica, vol. 45, no. 10, pp. 2292-2299, Oct. 2009.

[48] J. P. Gauthier, H. Hammouri, and S. Othman, "A simple observer for nonlinear systems - applications to bioreactors," IEEE Trans. Automat. Control, vol. 37, no. 6, pp. 875-880, Jun. 1992.

[49] R. Hermann and A. J. Krener, "Nonlinear controllability and observability," IEEE Trans. Automat. Control, vol. AC-22, no. 5, pp. 728-740, Oct. 1977.

[50] H. Fang, X. Zhao, Y. Wang, Z. Sahinoglu, T. Wada, S. Hara, and R. A. de Callafon, "Improved adaptive state-of-charge estimation for batteries using a multi-model approach," J. of Power Sources, vol. 254C, pp. 258-267, May 2014.

[51] Y. Wang, "Nonlinear geometric observer design," Ph.D. dissertation, University of Alberta, 2008

[52] G. Bornard and H. Hammouri, "A high gain observer for a class of uniformly observable systems," in Proc. 30th CDC, Brighton, England, 1991, pp. 1494-1496.

[53] H. Shim, Y. I. Son, and J. H. Seo, "Semi-global observer for multi-output nonlinear systems," Syst. Control Lett., vol. 42, no. 3, pp. 233-244, Mar. 2001.

[54] R. Marino, P. Tomei, and C. M. Verrelli, Induction Motor Control Design. London, UK: Springer, 2010.

[55] H. K. Khalil, Nonlinear Systems, 3rd ed. Englewood Cliffs, NJ: Prentice-Hall, 2002.

[56] K. Smith and C.-Y. Wang, "Solid-state diffusion limitations on pulse operation of a lithium ion cell for hybrid electric vehicles," J. of Power Sources, vol. 161, no. 1, pp. 628-639, 2006.

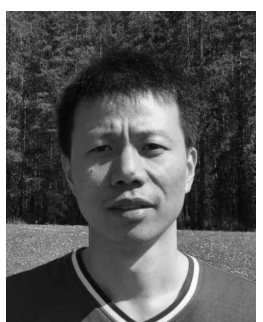

Yebin Wang received the B.Eng. degree in Mechatronics Engineering from Zhejiang University, China, in 1997, M.Eng. degree in Control Theory \& Control Engineering from Tsinghua University, China, in 2001, and Ph.D. in Electrical Engineering from the University of Alberta, Canada, in 2008. Dr. Wang has been with Mitsubishi Electric Research Laboratories in Cambridge, MA, USA, since 2009, and now is a Principal Member Research Staff. From 2001 to 2003 he was a Software Engineer, Project Manager, and R\&D Manager in industries, Beijing, China. His research interests include nonlinear control and estimation, optimal control, adaptive systems and their applications including mechatronic systems.

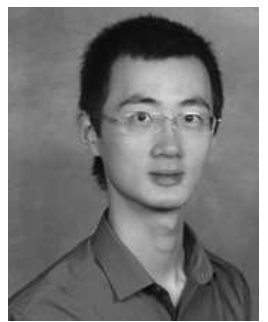

Huazhen Fang is an Assistant Professor in the Department of Mechanical Engineering, University of Kansas, Lawrence, KS. His research interest lies in control theory and its application to energy management and environmental monitoring. $\mathrm{He}$ earned his Ph.D. in Mechanical Engineering in 2014 from the Department of Mechanical \& Aerospace Engineering, University of California, San Diego. Prior to that, he received his M.Sc. in Mechanical Engineering from the University of Saskatchewan, Saskatoon, Canada in 2009, and B.Eng. in Compute Science \& Technology from Northwestern Polytechnic University, Xi'an, China in 2006. He held research intern positions at Mitsubishi Electric Research Laboratories and NEC Laboratories America in 2012 and 2013, respectively.

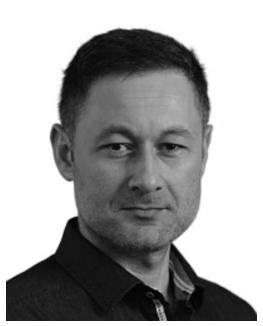

Zafer Sahinoglu received his MSc degree in 2001 and $\mathrm{PhD}$ in 2004 from New Jersey Institute of Technology in Biomedical Eng and Electrical Eng, respectively, and MBA from Massachusetts Institute of Technology Sloan School of Management in 2013. He worked at AT\&T's Shannon Research Labs as a visiting researcher in 2001, and then joined Mitsubishi Electric Research Labs. He has contributed and served in officer positions in numerous international standards in the areas of smartgrid, electric vehicles, indoor localization, wireless communications and sensor networks; and written two books published by Cambridge University Press. His current work includes remote sensing, robust modeling and optimization, battery modeling and energy big-data mining, and also development and integration of novel service businesses in productcentric companies.

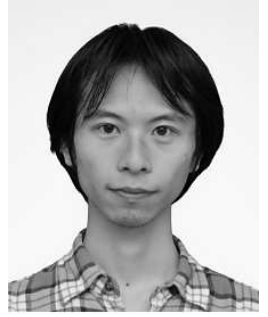

Toshihiro Wada received the B.Eng. degree in informatics and mathematical science and the $\mathrm{M}$ Informatics degree in Applied Analysis and Complex Dynamical Systems from the Kyoto University, Kyoto, Japan, in 2005 and 2007 respectively. His current research interests include sensing and optimization of battery systems.

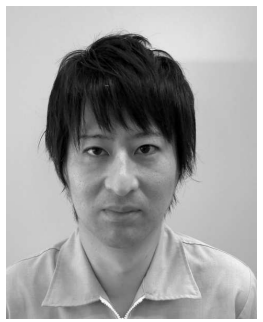

Satoshi Hara received the B.Eng. degree in electrical engineering from the Miyazaki University, Miyazaki, Japan and the M.S. degrees in electrochemistry from the Kyushu University, Fukuoka, Japan in 2008 and 2010 respectively. His current research interests include evaluation and analysis of lithium ion battery. 